\title{
Interstellar Extinction in the Vicinity of the Galactic Center
}

\author{
Angela S. Cotera \\ Jet Propulsion Laboratory ${ }^{1,2}$ \\ Janet P. Simpson ${ }^{2}$, Edwin F. Erickson, Sean W. J. Colgan \\ NASA/Ames Research Center \\ Michael G. Burton \\ University of New South Wales \\ and \\ David A. Allen ${ }^{3}$ \\ Anglo-Australian Observatory
}

Received __ ; accepted

${ }^{1}$ now at Steward Observatory, University of Arizona

${ }^{2}$ SETI Institute

${ }^{3}$ Deceased 


\begin{abstract}
We present $\mathrm{J}(1.2 \mu \mathrm{m}), \mathrm{H}(1.6 \mu \mathrm{m}), \mathrm{K}^{\prime}(2.11 \mu \mathrm{m})$ and $\operatorname{Br} \gamma(2.166 \mu \mathrm{m})$ images from four large regions within the central $40 \mathrm{pc}$ of the Galaxy. Localized variations in the extinction, as determined by observations of the stellar population, are examined using the median $\mathrm{H}-\mathrm{K}^{\prime}$ color as a function of position within each region. As the value of the derived extinction from the stars is dependent upon the intrinsic magnitude of the assumed stellar type, the $\mathrm{J}-\mathrm{H}$ vs. $\mathrm{H}-\mathrm{K}^{\prime}$ diagrams are first used to investigate the distribution of stellar types in the four regions. We find that there is a distinct OB population, contrary to earlier assumptions, with the ratio of $\mathrm{K}$ and $\mathrm{M}$ giants and supergiants to OB stars approximately twice that of the solar neighborhood. Although the on the scale of $\sim 1^{\prime}$ fluctuations in the extinction are on the order of $A_{V} \sim 2$, throughout the entire region the extinction varies from $A_{V} \gtrsim 25$ to $A_{V} \lesssim 40$. We also examine whether there is any variation in the extinction and stellar number density relative to the unsual radio features in these regions and do not find a significant correlation.

Spectral imaging in $\operatorname{Br} \gamma 2.166 \mu \mathrm{m}$ emission shows a strong morphological correspondence between the $6 \mathrm{~cm}$ radio images and the diffuse $\mathrm{Br} \gamma$ emission. By comparing the theoretical Br $\gamma$ flux derived from the radio flux using recombination theory, with our measured $\operatorname{Br} \gamma$ flux, we obtain a second, independent, estimate of the extinction. We compare the two data sets and find that the extinction as derived from the stars is consistently greater, sometimes by a factor of two, than the value of the extinction derived from the Bry images. The derived extinction in various regions, however, is insufficient for any of these regions to be located behind the molecular clouds as previously observed in the Galactic Center, consistent with the theory that the observed radio emission is
\end{abstract}


produced on the foreground surface of these clouds.

Subject headings: Galaxy: center-interstellar extinction 


\section{Introduction}

The Galactic Center (GC) is unobservable at visible wavelengths because of interstellar extinction, usually assumed to be $A_{V} \sim 30$, but known to vary between $5 \lessgtr A_{V} \lesssim 60$ (Catchpole, Whitelock \& Glass 1990). The bulk of the extinction is believed to be the result of clouds of dust and gas within $4 \mathrm{kpc}$ of earth and not due to clouds within the inner few hundred parsecs of the Galaxy (Sanders, Scoville \& Solomon 1985). Previous work to quantify the extinction towards the $\mathrm{GC}$ went to a limiting magnitude of $\mathrm{K}=12.1$, $\mathrm{H}=12.8$ and $\mathrm{J}=13.5$ (Catchpole et al. 1990), which, as shall be shown in $\S 5$, introduces a bias in the derived extinction. Other photometric studies in the GC have gone to fainter magnitudes, but to date these studies have been limited to the inner few parsecs (e.g. Blum et al. 1996). This work therefore removes the sample bias of previous studies and provides an examination of the variation in extinction and stellar population within $\lesssim 5^{\prime}$ of Sgr A. In addition, this study examines whether or not there is a correlation between variations in the extinction and the position of many of the unusual radio continuum features which make the inner $\sim 40 \mathrm{pc}$ of the Galaxy so intriguing.

Prior determinations of the large scale extinction variations derived from stellar colors have begun with the assumption that the stars observed in the GC are predominately evolved $\mathrm{K}$ and $\mathrm{M}$ giants. The validity of this assumption has been questioned for some time, at least since Lebofsky, Rieke \& Tokunaga (1982) first suggested that they were observing $\mathrm{M}$ supergiants in the GC and proposed there had been a recent burst of star formation. Several of their M supergiant candidates were subsequently shown to be M giants (Sellgren et al. 1987), but IRS 7 was confirmed to be a young M supergiant (Rieke \& Rieke 1989). Therefore the assumption deemed most appropriate was one which included a population of $\mathrm{M}$ giants and supergiants. That this is not the case, however, was suspected when young Of star candidates were identified within the central parsec (e.g. Forrest et al. 1989; Allen, 
Hyland \& Hillier 1990; Krabbe et al. 1991). When additional young massive WN and OB stars outside of the central parsec were unequivocally identified by their spectra (e.g. Cotera et al. 1996 (Paper I); Figer, McLean \& Morris 1996), the assumption of a population of exclusively $\mathrm{K}$ and $\mathrm{M}$ giants and supergiants was no longer valid. Recently, larger scale NIR observations of the central parsecs have confirmed that there is a luminous young population throughout the central 30 pcs (Mezger et al. 1999, Philipp et al. 1999)

With the discoveries of $\mathrm{O}, \mathrm{B}$ and $\mathrm{WN}$ star candidates at several locations within the central $\sim 40$ pc (e.g. Cotera et al. 1994; Nagata et al. 1995; Figer, McLean \& Morris 1995; Blum, DePoy \& Sellgren 1995; Paper I; Cotera et al. 1999 (Paper II)), in addition to a renewed interest in the general character of the stellar populations in the GC (Mezger et al. 1999, Philipp et al. 1999), there has also been a renewed effort to determine the intrinsic stellar properties of these stars (e.g. Najarro et al. 1997). In order to accurately address these and other areas of GC research, a good understanding of the extinction is necessary. We have a degenerate problem however: in order to use the infrared color-magnitude diagrams to determine the extinction, we must assume an intrinsic stellar type- but which stellar type to use is no longer obvious. Therefore, before we can determine the extinction from the $\mathrm{H}-\mathrm{K}^{\prime}$ colors, in $\S 3.2$ we use color-color diagrams to estimate a realistic ratio of $\mathrm{K}$ and $M$ giants and supergiants to $O B$ stars, which we then employ in our estimate of the extinction.

In addition to the photometric data, we have used the difference in the observed Br $\gamma$ $(2.166 \mu \mathrm{m})$ emission from several radio continuum regions, to that predicted from Case $\mathrm{B}$ recombination theory to derive a value of the extinction specifically for these features. Comparison shows that the extinction derived from radio recombination is up to a factor of 2 less than that derived from the stellar photometry. The $\operatorname{Br} \gamma$ emission images with their resultant extinction estimates are presented in $\S 4$. In $\S 5$ we discuss the implications of the 
two data sets taken together and argue that the radio emission must be from the foreground surface of the molecular clouds in the region and the stars must also be in front of these clouds.

\section{Observations and Data Reduction}

Observations were made at the Anglo-Australian Observatory on 1992 July 13-14 and 1993 June 13-15 with the facility Infrared Imaging Spectrometer (IRIS) which utilizes a Rockwell $128 \times 128$ pixel HgCdTe (NICMOS II) array. The $0.6^{\prime \prime} /$ pixel scale was selected for all observations. IRIS uses the $\mathrm{K}^{\prime}$ filter (central wavelength $2.1 \mu \mathrm{m}$; Wainscoat \& Cowie 1992), and standard $\mathrm{H}(1.6 \mu \mathrm{m})$ and $\mathrm{J}(1.2 \mu \mathrm{m})$ filters for broad band observations (differences in the photometric measurements of $\mathrm{K}$ and $\mathrm{K}^{\prime}$ are briefly discussed in $\S 3.1$ ). All data were linearized, bias subtracted, flat fielded and sky-subtracted with the astronomical software reduction package FIGARO.

We obtained $\mathrm{J}, \mathrm{H}$ and $\mathrm{K}^{\prime}$ images in four regions shown as shaded areas in Figure 1. The four regions are: 1) a portion of the Straight Filaments including AFGL 2004 (the 'Quintuplet cluster'), G0.15-0.04 (the 'Pistol ') and G0.18-0.04 (the 'Sickle', Yusef-Zadeh \& Morris 1987b), hereafter referred to as QPS; 2) the Arched Filaments including the E1 \& E2 Filaments and G0.10+0.02, hereafter referred to as the E1 \& E2 Filaments, (nomenclature from Morris \& Yusef-Zadeh 1989); 3) the region south of the Arched Filaments surrounding the radio emission regions H1-H8 (nomenclature Yusef-Zadeh \& Morris 1987a); and 4) the radio emission regions A-D just east of Sgr A East (Yusef-Zadeh \& Morris 1987a), which Zhao et al. (1993) call the Sgr A East H II cluster, hereafter referred to as Sgr A East. A combined broad band $\mathrm{J}, \mathrm{H}$ and $\mathrm{K}^{\prime}$ image of the E1 \& E2 Filaments was presented as Figure 1 of Paper I, the remaining three images are presented as Figures 2-4 overlaid with the available $6 \mathrm{~cm}$ radio contour maps. The figures are mosaics of $70^{\prime \prime} \times 70^{\prime \prime} \mathrm{J}, \mathrm{H}$, and $\mathrm{K}^{\prime}$ 
images which are combined in a three-color composite. These images were obtained during generally good conditions, with the image of QPS obtained on a night of $\lesssim 0.8^{\prime \prime}$ seeing. For the other images, the seeing was $\sim 1.0-1.5^{\prime \prime}$. Astrometry was performed using SAO stars within the large images. In most of the images, however, only two SAO stars were available which did not allow us to simultaneously constrain the three free parameters: RA plate scale, Dec plate scale and rotation angle. Therefore, on the QPS, E1 and E2 Filaments, and $\mathrm{H} 1-\mathrm{H} 8$ images, positions are accurate to $\$ 2.5^{\prime \prime}$. On the image of Sgr A East, optical astrometry was available for enough of the stars that the positions are good to $\sim 1^{\prime \prime}$.

Photometry results for all of the images were obtained using the crowded field procedures suggested for use with IRAF/DAOPHOT. Photometry was performed on the individual frames rather than the large images, to improve accuracy and provide a means of determining the errors in the derived magnitudes from the overlapping regions. Each individual $128 \times 128$ pixel image is extremely crowded; for instance, in each of the $70^{\prime \prime} \times 70^{\prime \prime}$ images at $K^{\prime}$ there are $Z 400$ stars. This crowding, the result of the large number of stars we observe while looking through the galactic plane, is the primary cause of the derived errors in magnitudes. Photometric errors are discussed in detail in $\S 3.1$. Only one frame per wavelength was taken at each position, so only one measurement is available for most stars, which contributes to the uncertainty: the magnitude error is thus 0.16 in $\mathrm{K}^{\prime}$ for the worst case, although the typical errors are $\lesssim 0.1$.

Our sensitivity for these observations varies slightly for each region as the regions were taken on different nights and in some cases different years. Plots of the log of the number of stars as a function of magnitude for each of the four regions are presented in Figure 5. Since we are dealing with a combination of disk and bulge stars, we know from Cohen (1993), that in the magnitude range we are observing, the $\log \mathrm{N}$ vs. magnitude is a relatively smoothly increasing function with decreasing magnitude. Therefore we fit a second order polynomial 
to the regions of the plot unaffected by either saturation or detection limits: in essence the well sampled regions of the plot. In order to determine the limiting magnitudes first we determined the standard deviation $(\sigma)$ of the fit in the well sampled region. We then used the point at which the data deviated from the fit by $\gtrsim 5 \sigma$ as the limiting magnitude in $\mathrm{H}$ and $\mathrm{K}^{\prime}$ and $z 3 \sigma$ for $\mathrm{J}$ as the fewer number of stars detected in $\mathrm{J}$ increased the noise in the fit. The values for $\mathrm{J}, \mathrm{H}$ and $\mathrm{K}^{\prime}$ determined in this way for each of the regions are presented in Table 1. The determination of the limiting magnitude at $\mathrm{K}^{\prime}$ in Sgr A East is effected by the significant noise in the region used for the fit due to the smaller sample, therefore the limiting $K^{\prime}$ magnitude presented in Table 1 is taken to be $3 \sigma$. In addition to the limiting magnitudes, Figure 5 illustrates the variations in the number of stars detected in the different regions. The E1 \& E2 Filaments and H1-H8 have approximately the same numbers of stars, which follows as they cover similar areas on the sky, and were taken in similar conditions. The data set around Sgr A East has considerably fewer stars due to the fact that only a $\sim 3^{\prime} \times 3^{\prime}$ region is included as opposed to $\sim 4^{\prime} \times 8^{\prime}$ in the E1 \& E2 Filaments. There are significantly more stars detected in QPS due to the excellent seeing during the night of 14 July 1992 and notably lower limiting magnitudes in $\mathrm{H}$ and $\mathrm{K}^{\prime}$.

Spectroscopic observations of the Pistol, a part of the Sickle, G0.10+0.02, part of the E2 Filament, H1, H2, H5-H7, H8, and Sgr A East regions A-D utilized data cubes, with axes $\alpha, \delta$ and $\lambda$. The data cubes were obtained by observing in spectral mode with a $70^{\prime \prime} \times 2.0^{\prime \prime}$ slit (resolution $\mathrm{R} \sim 250$ ) at fixed right ascension while the telescope stepped in declination at a rate of 36 "/hr, producing pixels which are approximately square in $\alpha$ and $\delta$ with a $0.6^{\prime \prime} /$ pixel plate scale. The cubes were flat fielded, straightened and had the atmosphere removed by dividing by a main sequence $G$ standard, generally taken before and after each data cube was obtained. Spectra of main-sequence early G standards were used for all flux calibrations (Allen \& Craig 1983). The flux calibration routine assumes the star radiates as a black body; a temperature of $5600 \mathrm{~K}$ was used for all $\mathrm{G}$ standards (Allen, 
D. A. 1976). Spectral images of each source were then extracted from the data cubes by

coadding the desired $\alpha, \delta$ planes (usually 3 planes centered on the emission wavelength) and using adjacent planes, containing no emission line features, for sky continuum subtraction.

Since the steps in declination used to build up the data cubes were not precisely $0.60^{\prime \prime}$, a result of the limits of telescope pointing accuracy, and there was some drift in RA over the often hour long integrations, the images extracted from the cubes are slightly irregular in RA and Dec. Therefore, the radio map overlays used in all figures do not always correspond precisely to the $\mathrm{Br} \gamma$ emission images. In addition, since the astrometry of the stellar images is only good to $\sim 2.5^{\prime \prime}$, and there is a very strong morphological correspondence between the radio continuum and the $\operatorname{Br} \gamma$ emission, the images which include $\operatorname{Br} \gamma$ emission use the correspondence between the radio continuum and the $\operatorname{Br} \gamma$ emission to establish positions which then are accurate to $\lesssim 1^{\prime \prime}$. Continuum images were also extracted from the cubes to determine the position of stars relative to the Br $\gamma$ emission and the broad band $\mathrm{H}$ and $\mathrm{K}^{\prime}$ images were then overlaid using these stellar positions. Due to the irregular pixel size in the cubes, the positions of the stars as seen in the continuum images extracted from the cube do not always correspond exactly with the broad band $\mathrm{H}$ and $\mathrm{K}^{\prime}$ images.

\section{Photometry}

\section{1. $\quad \mathrm{K}$ vs. $\mathrm{K}^{\prime} \&$ Errors}

IRIS utilizes the $\mathrm{K}^{\prime}$ filter $(2.11 \mu \mathrm{m})$ rather than the traditional $\mathrm{K}$ filter $(2.2 \mu \mathrm{m})$. The $\mathrm{K}^{\prime}$ band as described by Wainscoat \& Cowie (1992), was selected because it reduces the thermal component of the background allowing for deeper imaging in the same integration time. In Wainscoat \& Cowie (1992), they derive both empirical and observational relations for the conversion from $K^{\prime}$ to $K$, of the form 


$$
K^{\prime}-K=\alpha(H-K)
$$

where $\alpha$ needs to be determined for out specific observations. Wainscoat \& Cowie (1992) derive a value for $\alpha$ of 0.19 using the central wavelengths of the different bands, and $0.22 \pm 0.04$ based on observations of nearby stars; when they include two highly reddened stars $\alpha=0.18 \pm 0.04$. For our case, although additional observational calibration would be best, the value which includes the reddened stars is the appropriate choice. One additional problem, however, is the fact that the observationally calibrated value was based on observations performed at Mauna Kea while our observations were taken at the AAT.

In order to assess the effect on the derived relation due to the difference in observatory locations, we have used the atmospheric transmission code ATRAN (Lord 1992) to simulate the atmospheric conditions at the two sites. We have convolved these atmospheric models with the filter functions for $\mathrm{H}, \mathrm{K}$ and $\mathrm{K}^{\prime}$. Additionally, in order to investigate any differences which might arise from stellar type and extinction, we have further convolved the models with hot and cool blackbody spectra and the extinction curve of He et al. (1995). We find no theoretical dependence based on stellar type or extinction, but there is a correct required due to atmospheric differences: going from the AAT and Mauna Kea we find $\delta \alpha=0.013 \pm 0.001$. Therefore, adjusting the most appropriate value from Wainscoat \& Cowie (1992) to the AAT, we use $\alpha=0.17 \pm 0.04$ as the most appropriate value, throughout this paper. We note, however, that absolute values for $\alpha$ derived in the analysis based on the blackbody curves, were lower than 0.17 , which indicates that the values of $K^{\prime}$, and quantities derived using this conversion may be overestimates. The value used throughout this paper for $\alpha$ represents what we believe to be the best estimate possible, given the various constraints.

In determining the extinction to our sources, we use the extinction law of He et al. 
(1995) which we analytically transpose to $K^{\prime}$ using equation (1) and our derived value for $\alpha$. We adopt the value $E_{K^{\prime}-V} / E_{B-V}=-2.72$ throughout this paper.

Although the DAOPHOT routine assigns errors to each of the stars based on the variations in the background and fit to the PSF determined for each individual frame from stars in that frame, in regions of high confusion such as the $\mathrm{GC}$, it is advisable to make an independent determination of the errors by comparing the values derived for the same stars in different frames. We note that the largest errors are seen in the QPS data. On the night that the QPS data was taken, the seeing was excellent, $\lesssim 0.8^{\prime \prime}$. Since our pixel size is $\sim 0.6^{\prime \prime} /$ pixel, the data for QPS were undersampled, which results in larger errors. The difficulty of DAOPHOT in fitting stars with a PSF taken from undersampled data is reflected in errors derived by DAOPHOT which are approximately twice as large for the QPS stars as for the stars in all the other regions. Each of the images (Figures 2-4) consists of a mosaic of $70^{\prime \prime} \times 70^{\prime \prime}$ images. These individual frames overlap the adjacent images along their edges by $\sim 18^{\prime \prime}$ ( $\sim 30$ pixels). We therefore used stars detected in two images, as well as those in the corners where four frames overlapped, to obtain additional estimates of the photometry errors. One caveat to this approach is that as we transition from examining all stars detected, to looking only at stars found in two overlapping frames, to using only stars found in four overlapping frames, fewer and fewer stars are examined such that at some point low numbers statistics must be considered. In the large mosaic images (QPS, E1 and E2 Filaments, and $\mathrm{H} 1-\mathrm{H} 8$ ) there were $z 1000$ stars detected in both $\mathrm{H}$ and $\mathrm{K}^{\prime}$ in regions where two frames overlapped and $z 300$ stars found in $J, H$, and $K^{\prime}$ for the same regions. In regions where four frames overlapped, however, $\sim 65-100$ stars were detected in $\mathrm{H}$ and $\mathrm{K}^{\prime}$, and only 13-23 for stars found in $\mathrm{J}, \mathrm{H}$ and $\mathrm{K}^{\prime}$.

The median error as a function of magnitude for all of the images, using the errors determined from stars found in two overlapping frames, is shown in Figure 6. As expected, 
the error increases as we go to fainter magnitudes. The median error was selected in the following analysis, as opposed to the mean, to reduce the influence of random outliers. In addition to taking 2 or 4 overlapping frames to determine the errors, three separate cases, labeled in column 1 of Tables $2 a$ and $2 b$, were investigated according to the data to be presented. A: stars identified in both the $\mathrm{H}$ and $\mathrm{K}^{\prime}$ bands. $\mathbf{B}$ : stars found in both the $\mathrm{J}$ and $\mathrm{K}^{\prime}$ bands. $\mathrm{C}$ : stars which appear at all three wavelengths. The median error determined for the four large images for various cases are presented in Tables $2 \mathrm{a}$ and $2 \mathrm{~b}$. As the table shows, limiting the stars used for analysis to those found in $J, H$, and $\mathrm{K}^{\prime}$, generally improves the errors for the stars in $\mathrm{H}$ and $\mathrm{K}^{\prime}$ as the selection criteria eliminates the fainter stars which typically have larger errors. This is consist with the results presented in Figure 6. Also notable in Table 2a, the error determined using four measurements of the same star (cases $4 \mathrm{~A}-4 \mathrm{C}$ ) is $\sim 0.01-0.06$ magnitudes larger than those determined from only two overlapping frames. This could be a result of the much smaller sample size available to determine the median error in the case of stars with four measured magnitudes (e.g., in the QPS there are 1659 stars in case $2 \mathrm{~A}$ and only 78 in case $4 \mathrm{~A}$ ). The median error for the same stars as determined by DAOPHOT are also given in Tables $2 \mathrm{a}$ and $2 \mathrm{~b}$. The errors determined by DAOPHOT are consistently lower than those determined by comparing the same stars in different frames. In all subsequent analysis we use the errors as determined from two overlapping frames for the appropriate situation (e.g. case $\mathrm{C}$ for the $\mathrm{J}-\mathrm{H}$ vs. $\mathrm{H}-\mathrm{K}^{\prime}$ plot of QPS), as they provide a more conservative error than those derived by DAOPHOT, and do not suffer from the small number statistics such as those determined from four overlapping frames. The specific case used to establish the error bars is noted on all plots and corresponds to the values in Tables $2 \mathrm{a}$ and $2 \mathrm{~b}$. 


\subsection{Color-Color Diagrams}

The fundamental problem of using broad band colors to determine the interstellar extinction in regions of high extinction is having two independent color parameters to constrain: the intrinsic colors of the stars, and the color due to interstellar extinction. Previously (e.g. Catchpole et al. 1990), the assumption has been that since the population of stars in the Bulge are old stars, no more $\mathrm{OB}$ stars can exist in the GC and thus the population is assumed to be mixture of $\mathrm{K}$ and $\mathrm{M}$ giant and supergiants (which we will call simply KM stars). Recently however, NIR spectroscopy of GC stars has demonstrated that there are hot young stars within the central $\sim 50$ pc of the Galaxy (e.g. Krabbe et al. 1991, Cotera et al. 1994; Figer et al. 1995; Blum, et al. 1995, Paper I, Paper II). Therefore we must use the available data to examine the assumption of a strictly KM star population and attempt to derive a more realistic determination of actual stellar population.

We begin to constrain the intrinsic color of stellar population at the Galactic Center by noting that the only stellar types which can be detected at a distance of $8.0 \mathrm{kpc}$ (Reid 1993), assuming a minumum of $A_{V}=20$ and using our observational limits, are stars with intrinsic $\mathrm{M}_{J} \lesssim-2.0$. For reference, the intrinsic magnitudes of numerous stellar types are presented in Table 3. Using the derived limit, we immediately see that we are observationally restricted to most types of $\mathrm{OB}$ stars (including those on the main sequence), all $\mathrm{M}$ giants and all $\mathrm{K}$ and $M$ supergiants. However, the spectral types of $K$ giants which can be detected in the $\mathrm{J}$ bands drops sharply as a function of increasing $A_{V}$. For $A_{V} \lesssim 18$, we can observe all $K$ Giants to $K 0$, at $A_{V} \sim 20$, we can no longer detect spectral type $K 0$, and at $A_{V} \sim 25$, we can no longer detect any $\mathrm{K}$ giants. Therefore, if we use the $\mathrm{J}$ band data in determining our extinction values, our results would be skewed towards foreground stars. Therefore, we will use only the $\mathrm{H}-\mathrm{K}^{\prime}$ color to determine the interstellar extinction. As we noted above, 
however, we have two color parameters to determine and it is impossible to constrain two parameters with only one available color. We are therefore forced to use the $\mathrm{J}$ band data, in conjunction with the $\mathrm{H}$ and $\mathrm{K}^{\prime}$ data, to explore the relative stellar populations. By using the $\mathrm{J}$ band data, we are limiting the completeness of our sample; however, the assumption implied by this approach - the stellar population as measured by observations in $\mathrm{J}, \mathrm{H}$ and $\mathrm{K}^{\prime}$ is approximately equivalent to the actual population - is much better than the previous assumption of no OB stars. Therefore, although there are limitations to the method, it is the best possible solution given the constraints.

The $\mathrm{J}-\mathrm{H}$ vs. $\mathrm{H}-\mathrm{K}^{\prime}$ color-color diagrams for the four regions are presented in Figures 7-8. On each plot we have included the extinction curve for KM and OB stars, using the extinction curve of He et al. (1995) where $\mathrm{E}_{H-V} / \mathrm{E}_{B-V}=-2.76, \mathrm{E}_{J-V} / \mathrm{E}_{B-V}=-2.25$ and $E_{K^{\prime}-V} / E_{B-V}=-2.72$ as explained above. Using the appropriate errors for the specific data set, we have also plotted $1 \sigma$ error bars for the extinction curves, which are shown as shaded regions (see Tables $2 \mathrm{a}-2 \mathrm{~b}$ for exact error values). The intrinsic magnitudes used as the initial point for the two lines are determined by using the average of intrinsic colors for the two different population groups based on Table 3, with $\mathrm{M}_{K}$ converted to $\mathrm{M}_{K^{\prime}}$ using eguation (1). Throughout the remainder of this paper for the intrinsic colors of $\mathrm{OB}$ and KM stars we use: ${\overline{\left(J_{i}-H_{i}\right)}}_{O B}=-0.097 \pm 0.033,{\overline{\left(J_{i}-H_{i}\right.}}_{K M}=0.71 \pm 0.15$, ${\overline{\left(H_{i}-K_{i}^{\prime}\right)_{O B}}}=-0.044 \pm 0.025,{\overline{\left(H_{i}-K_{i}^{\prime}\right)_{K M}}}=0.161 \pm 0.037$.

As can be seen in Figures 7 and 8, many of the stars lie along the KM curve; however, in all the images except Sgr A East, a substantial number of stars also lie along the OB curve. In order to quantify these qualitative results, we use the extinction curves of KM and $\mathrm{OB}$ stars to determine which star are contained within the various shaded regions in Figures 7-8 (the extinction curve plus the estimated $1 \sigma$ errors). Based on the location of a star within these error boxes, a spectral type of either $\mathrm{KM}$ or $\mathrm{OB}$ is assigned. As can 
be seen in Figure 7, in the QPS region some stars are located within the error box of both spectral types. No effort has been made to force these stars into one type or another, and the extent to which the samples overlap suggests that the results of the analysis should be treated with caution. Due to the incompleteness of the sample due to limits at J, these results should be treated as indicating trends only. Stars outside of the error boxes are assumed to have either infrared excess or blue excess and are eliminated from the sample.

In order to assess whether or not the scatter in the $\mathrm{J}-\mathrm{H}$ vs. $\mathrm{H}-\mathrm{K}^{\prime}$ plots is actually the result of sampling two distinct stellar populations or is simply the result of random photometric errors greater than the estimated values, we looked at the number of stars with infrared excess versus blue excess in relation to a strictly KM population and a two group $\mathrm{KM}$ and $\mathrm{OB}$ population. If the scatter we are seeing is due only to random errors, we would expect that there should be equal numbers falling outside the error boxes of the different populations. Looking at stars outside of the error box for the KM extinction curve only, we find there are 3.5-5.0 times as many stars with red excess as those with blue excess. If, however, we divide the population into KM stars and $\mathrm{OB}$ stars, and look at the number of stars with blue excess and red excess in comparison to a two group population, we find the ratio is in fact $\sim 1.0-1.2$, consistent with random errors. Therefore, the assumption that there are in fact two distinct stellar populations, $\mathrm{K}$ and $\mathrm{M}$ giants and supergiants, and $\mathrm{OB}$ stars, appears to be quantitatively and qualitatively the correct assumption.

One subject of interest to Galactic Center researchers is whether there is a difference between the stellar populations and the extinction (as derived from the stellar population), as a function of position on and off the thermal radio emission regions. We explore this question, but given the constraints necessary in using the $\mathrm{J}$ band data, note that the derived ratios should be treated as upper limits. For each of the $70^{\prime \prime} \times 70^{\prime \prime}$ images in the E1 \& E2 Filaments and QPS, it was determined whether the position of the frame was coincident 
with predominately thermal radio emission (the Filaments, Pistol or Sickle), non-thermal emission (the Straight Filaments) or did not correspond to any radio features. For the $\mathrm{H} 1-\mathrm{H} 8$ region, since there is no non-thermal emission, the regions were divided into the thermal emission, the southeast corner and the northwest corner. Since there are only 9 frames for Sgr A East, we looked at the data in columns of $\sim 1_{\mathrm{EW}}^{\prime} \times 3_{\mathrm{NS}}^{\prime}$. The results of this analysis are presented in Table 4, which gives the median $\mathrm{H}-\mathrm{K}^{\prime}$ and $\mathrm{J}-\mathrm{H}$ values, and the average number of stars per frame based on whether they are included on the $\mathrm{OB}$ or KM extinction curve. To check on the significance of any determined variations, we look at random spatial variations by performing the same analysis going from north to south in rows of $\sim 2^{\prime}$ (roughly the distance covered by two images).

In the QPS region, the extinction between the various regions shows that there is no significant increase in extinction on the thermal filaments as opposed to off the filaments, or on the straight filaments. Actually, the differences in extinction are more significant simply moving from north to south than any possibly induced by location on or off the radio regions. In addition, the value of the extinction is far too small for the stars to be interior to molecular clouds in the region as Simpson et al. (1997) estimated that the extinction due to the molecular cloud is $A_{V} \sim 70$. Therefore, we conclude that the extinction to the stars is not effected by the thermal or non-thermal Filaments. In the E1 \& E2 filaments, a similar conclusion is reached, although more variations in the extinction are seen. In Table 4, we see that the largest extinctions are found in those regions which are off any radio feature. In addition, we see a definite trend of increasing extinction going from north to south, with an overall increase of $\delta \mathrm{A}_{\mathrm{V}} \lesssim 5$. In $\mathrm{H} 1-\mathrm{H} 8$, once again we see little change with location on and off the thermal regions, but there is an increase of $A_{V} \gtrsim 4$ going from the southeast corner to the northwest corner. In Sgr A East, going from east to west, we see a decrease in the extinction, with a total maximum change in the extinction of $A_{V} \sim 5$. 
The average number of $\mathrm{OB}$ and KM stars per frame (with each frame being approximately a square arcminute), is also presented in Table 4 . These results are more interesting, but given the small numbers of stars which fit all the imposed constraints, they should be treated with extreme caution. The most interesting result is the total ratio of OB to KM stars in the different regions. From a compilation of studies of the stellar populations in the solar neighborhood, Robin \& Crézé (1986) found that to a limiting magnitude of $\mathrm{M}_{V}=-0.5$, which corresponds to our limiting $\mathrm{J}$ magnitudes for a minimum of 20 magnitudes of visual extinction and the intrinsic colors of the stars under consideration, the ratio of $\mathrm{OB} / \mathrm{KM}$ stars is $\sim 1.0 \pm 0.05$. In the $\mathrm{QPS}$ region we find $\mathrm{OB} / \mathrm{KM} \sim 0.66$ (estimated errors are \pm 0.05 for all regions), in the $\mathrm{E} 1$ \& $\mathrm{E} 2$ Filaments $\mathrm{OB} / \mathrm{KM} \sim 0.53$, in $\mathrm{H} 1-\mathrm{H} 8$, $\mathrm{OB} / \mathrm{KM} \sim 0.51$; but in Sgr A East, OB/KM 0.25, although the number of KM stars in Sgr A East is consistent with the other regions. In QPS, a region we know to have numerous OB stars from spectroscopic observations (Figer et al. 1996) in do we see an increase in the ratio of OB/KM stars, which indicates that although limited, this method does confirm previously known results. In addition, in all of the regions which contain a significant amount of thermal radio emission, we see a significant $\mathrm{OB}$ population relative to the $\mathrm{KM}$ population. Although none of the regions have the same ratio as the solar neighborhood, there is a significant number of $O B$ stars that must be taken into account when determining the intrinsic color of the overall stellar population. In the QPS region, a few additional trends are noteworthy; the ratio of $\mathrm{OB} / \mathrm{KM}$ stars coincident with the straight filaments is $\sim 0.4$, significantly lower than the other regions. On the other hand, in regions where there is no corresponding radio emission, $\mathrm{OB} / \mathrm{KM} \gtrless 1.0$, which is consistent with the findings of Figer et al. (1996), as most of the identified hot massive stars lie in the region between the Pistol and Sickle, off of the primary radio features.

Finally, we examine the average extinction in $A_{V}$ from Table 4 in the various regions by using the derived $\mathrm{H}-\mathrm{K}^{\prime}$ or $\mathrm{J}-\mathrm{H}$ values, the corresponding stellar type and the following 
equations:

$$
\begin{aligned}
\mathrm{A}_{V} & =\frac{1}{0.051}\left[\left(H-K^{\prime}\right)-\left(H_{i}-K_{i}^{\prime}\right)\right] \\
\mathrm{A}_{V} & =\frac{1}{0.104}\left[(J-H)-\left(J_{i}-H_{i}\right)\right]
\end{aligned}
$$

The constants are derived using the extinction curve of $\mathrm{He}$ et al. (1995), adapted to $\mathrm{K}^{\prime}$ using equation (1). Using the same intrinsic colors, $\left(H-K_{i}^{\prime}\right)$ and $\left(J_{i}-H_{i}\right)$, used before for Figures 7-8, we then derived the visual extinction. For example, for $\mathrm{H}-\mathrm{K}^{\prime} \sim 1.59$, the median value for $\mathrm{QPS}$, on the thermal filaments, for the $\mathrm{OB}$ stars we find that $\mathrm{A}_{\mathrm{V}} \sim 32$. In general we find that the visual extinction as determined from OB stars exceeds that determined by $K M$ stars by an average of $A_{V} \gtrsim 6$. When combined with the previous analysis which indicates the ratio of $\mathrm{OB} / \mathrm{KM}$ stars is $\sim 0.5$ in the regions observed, this results suggests that assuming the stars observed in the GC at NIR wavelengths are $\mathrm{K}$ and $\mathrm{M}$ giants and supergiants underestimates the visual extinction by $\sim 2-3$ magnitudes (see $\S 5$ ). In converting the average $H-K^{\prime}$ values to an extinction in $A_{V}$, one should therefore assume the intrinsic magnitudes are a combination of an approximately half as many OB stars as KM stars. Finally, the derived extinction from the median $\mathrm{J}-\mathrm{H}$, is consistent with that derived from the median $\mathrm{H}-\mathrm{K}^{\prime}$ to within $\mathrm{A}_{\mathrm{V}} \lesssim 0.7$.

\subsection{Color-Magnitude Diagrams}

We present the $\mathrm{K}^{\prime}$ vs. $\mathrm{J}-\mathrm{K}^{\prime}$ diagrams for all four regions in Figure 9, and the $\mathrm{K}^{\prime}$ vs. $\mathrm{H}-\mathrm{K}^{\prime}$ diagrams in Figure 10. The Giant-branch curve for the $\mathrm{J}-\mathrm{K}^{\prime}$ diagrams is taken from Tiede, Frogel \& Terndrup (1995) who found that for Baade's Window, the Giant-branch was best fit by:

$$
(J-K)=(2.00 \pm 0.05)-(0.113 \pm 0.005) K
$$

which was converted to $\mathrm{K}^{\prime}$ using equation (1). Consistent with previous observations of the Galactic Center stars, we see that the observed stars scatter along the Giant-branch 
curve, although at a variety of extinctions. The Giant-branch curve in Figure 10 was taken from observations of 47 Tuc extended to higher luminosities by the observations of stars in Baade's window (Catchpole et al. 1990).

When comparing Figures 9 and 10 a few things are notable. First, we see that the average extinction indicated using the $\mathrm{J}$ vs. $\mathrm{J}-\mathrm{K}^{\prime}$ data is consistently lower that than indicated by the $\mathrm{K}^{\prime}$ vs. $\mathrm{H}-\mathrm{K}^{\prime}$ diagram. This supports the assumption that the best estimate of the extinction to the stars in the field should be derived from the $\mathrm{H}$ and $\mathrm{K}^{\prime}$ data only to avoid observational bias. Second, in Figure 9, we note there is a paucity of stars between $A_{V} \sim 10-20$, especially noticeable in the QPS region where we have a larger sample. This suggests we are seeing a foreground population separated from the stars at the Galactic Center by intervening dust and gas, consistent with the proposed clouds within $4 \mathrm{kpc}$ of earth (Sanders, Scoville \& Solomon 1985).

\section{Spectral Imaging}

The spectral images extracted from the data cubes provide two important pieces of information: the location of emission line stars and an estimate of the extinction towards the sources in question. Three color images of using emission images derived from the data cubes are presented in Figures 11- 18. In the Figures $\mathrm{Br} \gamma$ is blue, $\mathrm{K}^{\prime}$ is red, $\mathrm{H}$ is green, and the contours are the $6 \mathrm{~cm}$ radio maps. The $\mathrm{K}^{\prime}$ and $\mathrm{H}$ band images are taken from the large mosaic images and aligned with $2.2 \mu \mathrm{m}$ continuum images extracted from the cube. Several of the emission line images show the location of hot stars, which appear as strong compact sources. The nature of these stars and their spectra were presented in Papers I and II.

The areas which were selected for observations using the wider slit, drift scanning method are regions where there is strong radio flux consistent with thermal emission as 
seen in the $6 \mathrm{~cm}$ radio maps (Yusef-Zadeh \& Morris 1987a,b; Morris \& Yusef-Zadeh 1989). Recombination theory predicts that there should be detectable $\operatorname{Br} \gamma 2.16 \mu \mathrm{m}$ emission in these regions. In addition, if hot young stars are located within these regions, their strong NIR emission lines are detectable as compact sources within the same data scan. From the positions of the various features in the IR continuum, radio and $\mathrm{Br} \gamma$ images, we are able to determine if there are exciting stars near or coincident with the radio emission regions, and whether or not such stars have extinction values consistent with that of the diffuse gas.

In order to estimate the extinction to these regions, we must first determine the theoretical flux at $\operatorname{Br} \gamma(2.16 \mu \mathrm{m}), F_{B r \gamma}^{\text {theoretical }}$. From Rubin (1968), we derive:

$$
F_{B r \gamma}^{\text {theoretical }}=89.26 \beta \alpha_{\mathrm{B}}\left(\frac{S_{\nu}}{\mathrm{Jy}}\right)\left(\frac{\nu}{\mathrm{GHz}}\right)^{0.1} T_{e}^{0.35} \mathrm{erg} \mathrm{cm}^{-2} \mathrm{~s}^{-1}
$$

where $\beta$ is ratio of $\operatorname{Br} \gamma$ photons to Lyman continuum photons, $\alpha_{B}$ is the Case $B$ recombination coefficient, $S_{\nu}$ is the measured radio flux at frequency $\nu$, and $T_{e}$ is the electron temperature. $\beta$ is calculated using the results for Case $B$ recombination from Storey \& Hummer (1995) and Hummer \& Storey (1987) for the assumed electron density and temperature in the Galactic Center (see below). From Rubin (1968) we have that $\alpha_{\mathrm{B}}=4.10 \times 10^{-10} \mathrm{~T}_{e}^{-0.8}$ which agrees at $\mathrm{T}_{\mathrm{e}}=10^{4}$ with the more recent results of Osterbrock (1989). The above equation is derived using the same approach as that of Doherty et al. (1994) for observations of ultracompact H II regions, and also produces results within $10 \%$ of those derived using the equation of Smith et al. (1995) for an electron density, $\mathrm{n}_{e}$, equal to $10^{4} \mathrm{~cm}^{-3}$ and $\mathrm{T}_{e}=10^{4} \mathrm{~K}$.

For the Galactic Center, typical values of $n_{e}$ and $T_{e}$ for the known thermal emission radio regions vary from region to region. For the Pistol and Sickle, typical $\mathrm{n}_{e}$ are $\sim 1000-3000$ $\mathrm{cm}^{-3}$ (Simpson et al.. 1997); for the regions $\mathrm{H} 1-\mathrm{H} 8, \mathrm{n}_{e}$ range from $\sim 400-3000 \mathrm{~cm}^{-3}$ (Zhao et al.. 1993). Since the calculated value of $\beta$ is very weakly dependent upon electron 
density (varying $\lesssim 2 \%$ for order of magnitude changes in the electron density), we will set $\mathrm{n}_{e}=1000 \mathrm{~cm}^{-3}$. There is also a range of calculated electron temperatures varying from 5500 $\mathrm{K}$ for the region G0.095+0.01 (Erickson et al.. 1991), to $6000 \mathrm{~K}$ for the Pistol and Sickle (Simpson et al.. 1997), to $4200 \mathrm{~K}$ for the region H2 (Zhao et al.. 1993). The calculated value of $\beta$ is more sensitive to temperature than density, varying by $\sim 25 \%$ with a change in temperature of $2500 \mathrm{~K}$, we therefore assume $T_{e}=6000 \mathrm{~K}$, to be consistent with previous GC calculations (see e.g. Morris \& Yusef-Zadeh 1989; Simpson et al. 1997). We therefore find the best estimate for $\beta$ is $0.0170 \pm 0.004$

Finally, for all of the subsequent calculations we use the $6 \mathrm{~cm}$ radio maps. Therefore, to convert from measured radio flux to predicted $\mathrm{Br} \gamma$ flux for our Galactic Center data, we find from equation (5) that:

$$
\mathrm{F}_{B r \gamma}^{\text {theoretical }}=1.42 \times 10^{-21}\left(\frac{\mathrm{S}_{\nu}}{\mathrm{mJy}}\right) \quad \mathrm{W} \mathrm{cm}^{-2}
$$

Using this equation, the extinction is then given by:

$$
F_{B r \gamma}^{o b s e r v e d}=F_{B r \gamma}^{\text {theoretical }} e^{-\tau_{B r \gamma}}
$$

To determine $F_{B r \gamma}^{o b s e r v e d}$, we summed over those regions of $\mathrm{Br} \gamma$ emission which are described in detail in the following sections. The results are presented in Table 5. Rather than rely on published flux values, we obtained the copies of the $6 \mathrm{~cm}$ VLA radio maps available at http://imagelib.ncsa.uiuc.edu/project/document/95.FY, in order to insure the closest correspondence possible between the areas summed over in both the radio and our $\mathrm{Br} \gamma$ images. Comparison of our derived values with those published for $\mathrm{H} 5(\mathrm{~W}), \mathrm{H} 1$ and $\mathrm{H} 2$ by Zhao et al. (1993), indicate our derived radio fluxes are correct to within 10\%, which results in an extinction error of $\pm 0.1 \mathrm{dex}$. The derived values for $\tau_{B r \gamma}$ are presented in Table 5. These values of $\tau_{B r \gamma}$ give the extinction directly to the radio emission regions, with no assumptions made as to galactic location or extinction curves. As such they provide a 
completely independent estimate of the extinction to the radio sources, which are believed to be at the GC (e.g. Yusef-Zadeh \& Morris 1987a). Comparisons with the extinction derived from the stellar population and discussion of the individual sources are presented below.

In addition to $\mathrm{Br} \gamma$, we detected $\mathrm{He} \mathrm{I}(2.058 \mu \mathrm{m})$ emission in almost every region. However, recent work by Shields (1993) and DePoy \& Shields (1994) on the use of the $\mathrm{He} \mathrm{I} / \mathrm{Br} \gamma$ ratio as a nebular diagnostic indicates that a single ratio value is found for significantly different nebular parameters; hence, unless the electron temperature and density are well known, use of this ratio to determine the temperature of the ionizing source was not particularly effective. In addition, there are problems properly subtracting the atmosphere at the $2.058 \mu \mathrm{m}$ line, especially during long drift scans, thereby further reducing the quality of any derived ratio. Finally, Shields (1993) found that the dust content and internal velocity distributions significantly effected the line ratio. Therefore, since the requisite detailed information is not known for the regions under consideration, and we are dealing with complex morphologies, rather than the more simple planetary nebulae investigated in DePoy \& Shields (1994), the $\mathrm{He} \mathrm{I} / \mathrm{Br} \gamma$ is not used in the analysis of these regions.

\subsection{Quintuplet, Pistol and Sickle}

Three data scans were taken in the QPS region; the one taken at the southern end of the Sickle did not show Bry emission significantly above our noise and therefore is not presented. In addition to the emission from the Pistol itself, the emission image of the Pistol revealed four emission line stars discussed in detail in Paper I. In the combined $\mathrm{Br} \gamma$ $\mathrm{H}, \mathrm{K}^{\prime}$, and radio image, presented in Figure 11, we clearly see that the diffuse emission 
from the Pistol closely follows the radio emission. Directly above the Pistol, there are two compact sources (numbers 8 and 10) which correspond to two stars that are part of a group of stars known alternately as AFGL 2004, or the Quintuplet Cluster after the five luminous cool stars (numbered 1, 2, 3, 4 and 9 in Figure 11), originally detected in $\mathrm{K}$ band images (e.g. Nagata et al. 1990). In addition to \#8 and \#10 directly above the Pistol, we see a compact source in the extreme upper left corner corner, corresponding to a star, which we call Source B. Directly below the Pistol we see a compact source, we call Source A in Paper I, which has recently been observed with HST (Figer et al. 1998), and is now known as the 'Pistol Star'. The Pistol Star is a single star which may be one of the most massive stars yet detected (Figer et al. 1998), and corresponds to a slight peak in the radio emission (Paper I). In comparison to the other stellar sources in our image it appears to be slightly extended. The spectra and discussion of the stars is presented in Paper I and is also discussed by Figer et al. (1996) and Morris et al. (1996). We also detected significant He I $2.058 \mu \mathrm{m}$ emission from the Pistol which follows the Br $\gamma$ emission. Notable in the He I image, however, is the lack of emission from the Pistol Star.

The Br $\gamma$ emission image of the Sickle (G0.18-0.04, Figure 12), is interesting for a number of reasons. As seen in the other images, the morphology of the $\mathrm{Br} y$ emission is similar to the radio emission, both of which are extremely clumpy. Although the emission comes from a series of compact knots, none of the knots correspond to stars in the continuum images. In addition, there are no emission stars detected in this scan although there may be additional OB stars nearby (see Figer et al. 1996). The Sickle is the only scan in this data set to cover portions of the Straight Filaments (see Figure 1) which are approximately constant in declination at scale, so would be parallel to the $\mathrm{x}$-axis in the image. No emission from the Straight Filaments is seen, consistent with the nonthermal nature of that emission. 


\subsection{E1 and E2 Filaments}

The radio emission region $\mathrm{G} 0.10+0.02$ is the site of the strongest $6 \mathrm{~cm}$ radio emission in the Arched Filaments and the radio map also shows several clumps of concentrated flux within the region. From the observed FIR emission lines Erickson et al. (1991) estimated that numerous $\mathrm{O}$ stars are needed to ionize the region. The $\mathrm{Br} \gamma$ emission image of the region combined with the $\mathrm{H}$ and $\mathrm{K}^{\prime}$ images and overlaid with the radio contours is shown in Figure 13. In the image we see that the diffuse emission is again similar to the radio emission. In the upper left region of the image, there is a compact $\operatorname{Br} \gamma$ emission source, labeled 'A', which is coincident with a star. The spectrum of the star is discussed in

detail in Paper II and appears to be either an extreme Of star or a late type WN star. As can be seen in Figure 13, however, no emission line stars are embedded within the radio region itself. The spectra of several of the stars lying interior to the bottom of G0.10+0.02 were extracted from the data cube of the region: none exhibit emission lines, and all had indications of $\mathrm{CO}$ absorption at $z 2.3 \mu \mathrm{m}$, indicating they are cooler $\mathrm{M}$ giants and therefore unlikely to be the ionization sources for the region.

Only one region north of G0.10+0.02 along the E2 Filament was observed with a spectral scan. The location of the scan, labeled "E2 Filament" in Figure 1, is at a peak in the $6 \mathrm{~cm}$ radio emission. The three color $\operatorname{Br} \gamma$ emission image is presented in Figure 14. Once again the $\operatorname{Br} \gamma$ emission has the same appearance as the radio emission; however, in the E2 Filament Br $\gamma$ image, no compact emission sources are seen. In both G0.10+0.02 and the E2 filaments, the He I emission images at $2.06 \mu \mathrm{m}$ were extracted from their respective data cubes but yielded little additional information as the flux was not significantly above the background. 


\subsection{Regions $\mathrm{H} 1-\mathrm{H} 8$}

H1-H8, a group of compact $\mathrm{H}$ II regions between Sgr A and the Arched Filaments, do not appear to be morphologically related to these regions other than by their apparent proximity. To date, little IR work has been done on the H1-H8, although they are believed to be in the GC (Yusef-Zadeh \& Morris 1987b; Zhao et al. 1993). In H1, presented in Figure 15 , we see once again see the similarity of the diffuse emission and the radio emission. Although there are stars within the region, none show significant line emission above the diffuse background level. There are stars located coincidentally along the arc of diffuse emission, but when compared to the stars found in the other images do not stand out. This is the only region in this study where the emission mapped in $\operatorname{Br} \gamma$ and the radio is also detected in the $\mathrm{K}^{\prime}$ image. In the extreme right of Figure 15, the $\mathrm{Br} \gamma$ emission corresponds to region $\mathrm{H} 2$ which is shown more clearly in Figure 16. In the $\mathrm{Br} \gamma$ image of the $\mathrm{H} 2$ region, the radio morphology is again reproduced; this is particularly apparent in the arc feature below the bright compact region which seems to connect to the $\mathrm{H} 2$ region. There are two compact emission features at the peak of the $\mathrm{H} 2$ emission, one of which is likely a $\mathrm{B}[\mathrm{e}]$ star and the other a Red Giant or Supergiant, both discussed in Paper II. At the peak of $\mathrm{H} 2$, neither the $\mathrm{Br} \gamma$ or the radio emission are perfectly spherical, but instead are slightly elliptical, with the major axis of the ellipse laying on an axis between the two stars (as can clearly be seen in Figure 16). Therefore, it seems likely that both stars are associated with the region $\mathrm{H} 2$.

Nearer to the Arched Filaments, observations were made of the region including the western section of $\mathrm{H} 5$, and all of $\mathrm{H} 6$ and $\mathrm{H} 7$. In the $\mathrm{Br} \gamma$ image (Figure 17), we see an interesting emission line star. The star, labeled 'A', is located to the east of the crescent

of emission from $\mathrm{H} 5$ and is nearly at the center of curvature for the entire H5 region (see Paper II, Figure 7). In Paper II we argue that based on both morphological and empirical 
grounds, this star is responsible for the ionization of H5. Also seen in Figure 17 is the emission from the nearby regions $\mathrm{H} 6$ and $\mathrm{H} 7$. The emission from $\mathrm{H} 6$ is extremely faint while the emission from $\mathrm{H} 7$ is more easily seen. It is most likely that $\mathrm{H} 7$ is a compact $\mathrm{H}$ II region, although in the continuum images there are two possible stars which may be associated with $\mathrm{H} 7$.

Finally, a drift scan was made of the region including $\mathrm{H} 8$ (Figure 18), and a star was determined to be located within the region. The spectrum of this star indicates it is very likely a $\mathrm{B}[\mathrm{e}]$ star, a more detailed discussion of its stellar type is presented in Paper II.

\subsection{Sgr A East, Regions A-D}

The radio emission to the east of Sgr A East, regions A-D (Figure 19), comes from sources which are much more compact and spherically symmetric than most of the previous regions. However, the regions appear to be slightly distorted in the direction of the Sgr A East. Yusef-Zadeh \& Morris (1987a) have suggested the curvature in the flux is due to interaction with the expanding SNR in Sgr A East. In the Bry emission image (Figure 19), we see both the emission from the probable H II regions, and a compact emission line star adjacent to region $\mathrm{A}$. This star is fascinating as it contains some of the strongest, broadest lines of any star seen in the area and is almost certainly a WN6 star (see Paper II). To the left (east) of region $\mathrm{D}$, we see two faint emission sources, which could be additional stars, however, results from the other images suggest that if the emission were stellar, it would be much stronger as compared to the diffuse emission. Therefore this faint emission may be the result of difficulties in background subtraction as the sky conditions varied over the 1.5 hour scan integration time. Finally, the regions A-D have the strongest He I $2.058 \mu \mathrm{m}$ emission of any of the sources. 


\section{Extinction}

As seen in $\S 3.3$, the inclusion of $\mathrm{J}$ band data introduces a bias towards foreground stars, therefore we use only the $\mathrm{H}$ and $\mathrm{K}^{\prime}$ data to derive the extinction to the regions which we will compare with the $\operatorname{Br} \gamma$ extinction. In addition, since we will be using the average value of $\mathrm{H}-\mathrm{K}^{\prime}$, we further restrict the sample to stars with $0.8 \lesssim \mathrm{H}-\mathrm{K}^{\prime} \lesssim 3.0$, to eliminate obvious foreground stars and to avoid the problem of incompleteness as we move to fainter values of $\mathrm{H}$. We also do not take stars with $\mathrm{H}$ below the limiting magnitude for the given regions as presented in Table 1. The average color values by frame are presented Figures $20-23$ overlaid on the radio contours. The error on the determined average color is $\sim 0.02$ dex.

The previous study of this kind in this region (Catchpole et al. 1990), had limiting magnitudes of $\mathrm{H}=12.8$ and $\mathrm{K}=12.1$ and assumed the stellar population was comprised only of $\mathrm{K}$ and $\mathrm{M}$ stars. With our limiting magnitudes of $\mathrm{K}^{\prime} \sim 14-15$ and $\mathrm{H} \sim 15-16$, we are observing 4-18 times more stars than the previous study. If, using our data sets, we recalculate the average colors imposing the limiting $\mathrm{H}$ magnitude of Catchpole et al.(12.8) we find that the derived extinction values decrease by $A_{V} \lesssim 1-4$ magnitudes (assuming a population of $\mathrm{K}$ and $\mathrm{M}$ stars only). Thus, if we restrict our data to the same population observed by Catchpole et al. we find the calculated average extinction values decreases by $15 \%$. However, we have argued $(\S 3.2)$ that the stellar population is a mixture of $\mathrm{OB}$ and KM stars. Assuming a mixed population we find the visual extinction given by Catchpole et al. is underestimated by $4-7$ magnitudes, or by $\sim 20 \%$.

In $§ 3.2$, we saw that, except for the region near Sgr A East, the stellar population is divided approximately into twice as many $\mathrm{K}$ and $\mathrm{M}$ giants and supergiants as $\mathrm{OB}$ stars. Therefore, to derive the extinction in $A_{K}$ from the numbers given in Figures 20-22, we use a weighted average value for these stellar types, $\overline{\left(H_{i}-K_{i}^{\prime}\right)}=0.11 \pm 0.01$, with $\mathrm{K}$ converted $K^{\prime}$ using equation (1). The derived values of $A_{K}$ for the regions studied using the $\operatorname{Br} \gamma$ 
maps are presented in Table 6. For the region Sgr A East, since the ratio of OB/KM stars $\sim 0.25$, and we have very few stars on which to make this assessment, we have assumed that the stars are best represented by $\mathrm{K}$ and $\mathrm{M}$ giants and supergiants, and therefore use ${\overline{\left(H_{i}-K_{i}^{\prime}\right)_{K M}}}_{K}=0.16$ to calculate $A_{K}$ for regions $\mathrm{A}-\mathrm{D}$.

As can be seen in Table 6 the values derived from the Bry studies, except in the regions $\mathrm{H} 1$ and H6, are consistenly lower than those derived by looking at the corresponding stellar populations, up to a factor of two in a few cases. One possible explanation for this is that the values of the radio flux taken from the VLA map are underestimated due to the losses inherent in interferometric observations. To check on this possibility, we have used the single dish $43 \mathrm{GHz}$ map of Sofue et al. (1986) to derive the radio flux to the two regions in this study which can be isolated in the $43 \mathrm{GHz}$ map: G0.10+0.02 and the Sickle. We find that for $\mathrm{G} 0.10+0.02$, from the $43 \mathrm{GHz}$ map $\tau_{\mathrm{Br} \gamma} \sim 2.6$ as compared to 2.1 from the $6 \mathrm{~cm}$ radio map. For the Sickle, we find that $\tau_{\mathrm{Br} \gamma}$ derived from the $43 \mathrm{GHz}$ map is the same as that derived from the $6 \mathrm{~cm}$ map. These comparisons, however, suffer from differences in beam size and an inability to accurately match corresponding regions in our NIR observations and the $43 \mathrm{GHZ}$ observations. Therefore, as a second check, we calculated the extinction we would derive from our $\operatorname{Br} \gamma$ data if $50 \%$ of the VLA emission was lost. The results of this comparison are listed in column 3 of Table 6 . By assuming that there is emission missing in the VLA radio observations, we can reconcile our estimates of the extinction derived from the measured Bry emission to that derived from the stellar population in the Pistol, Sickle, $\mathrm{H} 1, \mathrm{H} 5-\mathrm{H} 7$, and D.

The derived extinctions in regions $\mathrm{H} 2$ and $\mathrm{H} 8$, however, cannot be reconciled by assuming radio losses. This is most likely a result of the $\operatorname{Br} \gamma$ emission in these two regions having a strong stellar component. In these cases, it is not expected that Case B 
recombination theory would provide an accurate estimate of the $\operatorname{Br} \gamma$ emission (Thompson 1984). The fact that the extinction values in region $\mathrm{D}$ can be reconciled assuming only radio losses supports the idea that the emission lines seen coincident with a star at that location (Paper II) are nebular rather than stellar in origin since a strong stellar contribution would suggest a greater discrepancy in extinction estimates.

The remaining regions, G0.10+0.02, the peak of the E2 Filament, and $\mathrm{A}-\mathrm{C}$, are more difficult to understand. One possibility is that these regions are foreground objects. For G0.10+0.02 and the E2 peak, however, this is not a particularly good explanation as the Arched Filaments are apparently interacting with the Straight Filaments (Morris \& Yusef-Zadeh 1989), and the observed radio emission is likley on the surface of a dense molecular cloud which we would not anticipate penetrating (Serabyn \& Güsten 1987). In addition, the data from G0.10+002 and the peak of the E2 Filament have the lowest signal-to-noise data in the set, and the derived analytical errors may have underestimated the quality of the measurements. In the regions $\mathrm{A}-\mathrm{C}$, however, no such problem exists, and the possibility that these regions are in fact foreground objects cannot be ruled out by this analysis.

The primary conclusion that can be drawn from this comparison is that the ionized gas seen in the radio maps cannot be embedded within the coincident molecular clouds. The extinction within the $+25 \mathrm{~km} \mathrm{~s}^{-1}$ cloud, co-located with the Sickle, has been estimated to be $A_{V} \sim 70$ (Simpson et al. 1997), therefore no stars would be observable behind this cloud. In addition, if the ionized gas observed in the radio and IR were interior to this cloud, the extinction derived from the $\operatorname{Br} \gamma$ data, would be expected to be much larger, but again this is not the case. The observed ionized gas is therefore most likely on the front face of the molecular clouds as suggested earlier by Serabyn \& Güsten (1987). However, with the effects from possible underestimates of the VLA radio flux and the difficulties in matching 
the areas observed with the $43 \mathrm{GHz}$ map with the data presented here, determining whether the ionized gas is also in front of part of the stellar populations is problematic at best. Finally, given the potential errors in the extinction as estimated from a comparison of radio and $\operatorname{Br} \gamma$ data discussed above, the extinction value we derive from the stellar population appears to be the more consistent and reliable of the two methods.

\section{Conclusions}

There appear to be no correlations between the variations in the local stellar population and the location of the radio emission regions: there are no increases in stellar density with location on or off the radio regions, and no apparent effect in the average stellar extinction with location on the thermal emission regions. Studies of the stellar population indicate that the ratio of $\mathrm{OB}$ to $\mathrm{KM}$ stars is about half that of the solar neighborhood in several regions. This contradicts the assumption that all of the stars in the GC are evolved K and $M$ giants and supergiants, and provides further evidence that there has been recent star formation in this region. In addition, previous extinction estimates may be too low by $\sim 20 \%$ due to the limiting $\mathrm{H}$ and $\mathrm{K}$ magnitudes and the assumed stellar population. The derived extinction directly to the radio emission regions is in general, less than the stellar population indicating, at a minimum, that the emission seen from these regions in on the face of the observed molecular clouds, and some of the regions may be closer than the Galactic Center. In addition, the ionization of these regions is stellar in origin as many of the regions have either nearby, or embedded emission line stars.

We would like to acknowledge the considerable assistance of the Anglo-Australian Observatory staff in obtaining and reducing this data, and Lindsey Davis at NOAO for considerable help with DAOPHOT data reduction. We would also like to thank the 
anonymous referee for valuable suggestions on the use of the $\mathrm{K}^{\prime}$ filter and the $\mathrm{J}$ band data. ASC acknowledges the support of the National Research Council Associateship Program; JPS and SWJC acknowledge support of NASA/Ames Research Center Research Interchange grants $\mathrm{NCC} 2-900$ and $\mathrm{NCC} 2-647$ respectively. 


\section{REFERENCES}

Allen, C.W. 1976, Astrophysical Quantities, (London: The Athlone Press LTD)

Allen, D. A. 1976, MNRAS, 174, 29P

Allen, D. A. \& Craig, T. A. 1983, MNRAS, 203, 777

Allen, D. A., Hyland, A. R. \& Hillier, D. J. 1990, MNRAS, 244, 706

Bessel, M. S. \& Brett, J. M. 1988, PASP, 100, 1134

Blum, R. D., DePoy, D. L. \& Sellgren, K. 1995, ApJ, 441, 603

Blum, R. D., Sellgren, K., DePoy, D. L. 1996, ApJ, 470, 864

Catchpole, R. M., Whitelock, P. A., \& Glass, I. 1990, MNRAS, 247, 479

Cohen, M. 1993, AJ, 105, 1860

Cotera, A.S., Simpson, J.P., Erickson, E.F., Colgan, S.W.J., Burton, M.G, \& Allen, D.A. 1999, ApJ, 510, 747 (Paper II)

Cotera, A.S., Erickson, E.F., Allen, D.A., Simpson, J.P., Colgan, S.W.J., \& Burton, M.G. 1996, ApJ, 471, 750 (Paper I)

Cotera, A.S. 1995, Ph.D. Thesis, Stanford University

Cotera, A.S., Erickson, E.F., Allen, D.A., Simpson, J.P., Colgan, S.W.J. \& Burton, M.G., 1994 NATO Conference on Nuclei of Galaxies: Lessons Learned from the Galactic Center, ed. R. Genzel \& A. I. Harris, (Dordrecht: Kluwer Academic Publishers), p. 217

DePoy, D. L. \& Shields, J. C. 1994, ApJ, 422, 187

Doherty, R. M., Puxley, P., Doyon, R., Brand, P. W. J. L. 1994, MNRAS, 266, 497

Elias, J. H., Frogel, J. A, \& Humphreys, R. M. 1985, ApJS, 57, 91 
Erickson, E. F., Colgan, S. W. J., Simpson, J. P., Rubin, R. H., Morris, M. \& Haas, M. R. 1991, ApJ, 370, L69

Figer, D.F., McLean, I., \& Morris, M. 1995, ApJ, 447, L29

Figer, D.F., McLean, I.S. \& Morris, M. 1996, Proceedings of the 4th ESO/CTIO Workshop: "The Galactic Center", R. Gredel (ed.), ASP Conf. Ser. Vol. 102, p. 263

Figer, D.F., Najarro, F., Morris, M., McLean, I.S., Geballe, T.R., Ghez, A.M., Langer, N. 1998, ApJ, 506, 384

Forrest, W., Shure, M. A., Pipher, J. L. \& Woodward, C. E. 1987 in The Galactic Center, ed. D. C. Backer (New York:AIP), 153

Hummer, D. G., \& Storey, P. J. 1987, MNRAS, 224, 801

He, L., Whittet, D. C. B., Kilkenny, D., \& Spencer Jones, J. H. 1995, ApJS, 101, 335

Lebofsky, M. J., Rieke, G. H \& Tokunaga, A. T. 1982, ApJ, 263, 736

Lord, S. D. 1992, NASA Technical Memorandum 103957 (Moffett Field: NASA)

Mezger, P. G., Zylka, R., Philipp, S. \& Launhardt, R. 1999, A\&A, 348, 457

Morris, M., \& Yusef-Zadeh, F. 1989, ApJ, 343, 703

Morris, P., Eenens, P.R.J., Hanson, M.M., Conti, P.S. \& Blum, R.D. 1996 ApJ, 470, 597

Nagata, T. et al. 1990, ApJ, 351, 83

Nagata, T., Woodward, C. E., Shure, M. \& Kobayashi, N. 1995, AJ, 109, 1676

Najarro, F., Krabbe, A., Genzel, R., Lutz, D., Kudritzki, R. P., \& Hillier, D. J. 1997, A\&A, 325,700

Osterbrock, D. E. 1989, Astrophysiçs of Gaseous Nebulae and Active Galactic Nuclei, (Mill Valley Calf.: University Science Books). 
Philipp, S., Zylka, R., Mezger, P. G., Duschl, W. J., Herbst, T. \& Tuffs, R. J. 1999, A\&A, 348,768

Reid, M. J. 1993, ARA\&A, 31, 345

Robin, A., \& Crézé, M. 1986, A\&A, 157, 71

Rubin, R. H. 1968, ApJ, 154, 391

Sanders, D. B., Scoville, N. Z., \& Solomon, P. M. 1985, ApJ, 289, 373

Sellgren, K. Hall, D. N. B., Kleinmann, S. G., \& Scoville, N. Z. 1987, ApJ, 317, 881

Serabyn, E. \& Güsten, R. 1987, A\&A, 184, 133

Serabyn, E. \& Morris, M. 1994, ApJ, 424, L91

Shields, J. C. 1993, Ap.J. 419, 181

Simpson, J. P., Colgan, S. W. J., Cotera, A. S., Erickson, E. F., Haas, M. R., Morris, M. \& Rubin, R. H. 1997, ApJ, 487, 689

Smith, D. A., Herter, T., Haynes, M., Beichman, C. A., \& Gautier, T. N. 1995, ApJ, 439, 623

Sofue, Y., Inoue, M., Handa, T., Tsuboi, M. Hirabayashi, H., Morimoto, M., \& Akabane, K. 1986, PASJ, 38, 475

Storey P. J. \& Hummer, D. G. 1995, MNRAS, 272, 41

Thompson, R. I. 1984, ApJ, 283, 165

Tiede, G. P., Frogel, J. A., \& Terndrup, D. M. 1995, AJ, 110, 2788

Wainscoat, R. J. \& Cowie, L. L. 1992, AJ, 103, 332

Wegner, W. 1994, MNRAS, 270, 229

Yusef-Zadeh, F. \& Morris, M. 1987a, AJ, 94, 1178

Yusef-Zadeh, F. \& Morris, M. 1987b, ApJ, 320, 545 
Zhao, J. H., Desal, K., Goss, W. M., \& Yusef-Zadeh, F. 1993, ApJ, 418, 235 
Fig. 1.- Position of the infrared images presented in this paper overlaid with the $6 \mathrm{~cm}$ radio contours (Yusef-Zadeh \& Morris 1987a). The shaded areas indicate the regions where large broad band images were obtained. The labeled regions indicate radio regions also observed in $\operatorname{Br} \gamma$.

Fig. 2.- Three color $J, H, K^{\prime}$ image of the QPS region. $J$ is blue, $H$ is green and $K^{\prime}$ is red. The image is a mosaic of $2470^{\prime \prime} \times 70^{\prime \prime}$ images, obtained in a $4 \times 6$ array. Seeing for the image was $\lesssim 0.8^{\prime \prime}$.

Fig. 3.- Three color $J, H, K^{\prime}$ image of the $\mathrm{H} 1-\mathrm{H} 8$ region. $\mathrm{J}$ is blue, $\mathrm{H}$ is green and $\mathrm{K}^{\prime}$ is red. The image is a mosaic of $3070^{\prime \prime} \times 70^{\prime \prime}$ images, obtained in a $5 \times 6$ array.

Fig. 4.- Three color $\mathrm{J}, \mathrm{H}, \mathrm{K}^{\prime}$ image of the Sgr A East region. $\mathrm{J}$ is blue, $\mathrm{H}$ is green and $\mathrm{K}^{\prime}$ is red. The image is a mosaic of $970^{\prime \prime} \times 70^{\prime \prime}$ images, obtained in a $3 \times 3$ array. The enhancement of stars seen in the northwest corner, is the concentration of stars along the plane of the galaxy.

Fig. 5.- The log of the number of stars versus magnitude as detected in each of the four regions. As can be seen, many more stars were detected in the QPS region due to the subarcsec seeing. The smaller total number of stars in Sgr A East region is due the result of having taken fewer images of this region.

Fig. 6.- Median error in magnitude vs. median $\mathrm{K}$ magnitude. The largest errors are in the QPS frames, due to undersampling (see text). As expected, the error generally increases with increasing magnitude.

Fig. 7.- The Color-Color diagram for QPS and the E1 and E2 filaments. Errors bars are shown in the lower right corner along with the case used (see text for details and Table $2 \mathrm{a}$ for numbers). The shaded boxes are the estimated errors applied to the reference stellar 
extinction curves. Stars within these boxes are assumed to be of the same stellar type as the nearest spectral curve.

Fig. 8.- The Color-Color diagram for the regions H1-H8 and Sgr A East. Errors bars are shown in the lower right corner along with the case used (see Table $2 \mathrm{~b}$ ). The shaded boxes are the assumed errors applied to the reference stellar extinction curve.

Fig. 9.- The $\mathrm{K}^{\prime}$ vs. $\mathrm{J}-\mathrm{K}^{\prime}$ diagram for all the regions. Errors bars are shown in the lower right corner along with the case used (see Tables 2a-2b). The Giant-branch curve is from Tiede et al. (1995) and the extinction curve that of He et al. (1995) both reference curves have been adjusted to $K^{\prime}$ using equation (1).

Fig. 10.- The $\mathrm{K}^{\prime}$ vs. $\mathrm{H}-\mathrm{K}^{\prime}$ diagram for all the regions. Errors bars are shown in the lower right corner along with the case used (see Tables $2 \mathrm{a}-2 \mathrm{~b}$ ). The Giant-branch curve is from Catchpole et al. (1990) and the extinction curve that of He et al. (1995) both adapted to $\mathrm{K}^{\prime}$ using equation (1).

Fig. 11.- Three color image of G0.15-0.04, the 'Pistol'. $\mathrm{K}$ ' is red, $\mathrm{H}$ is green, $\mathrm{Br} \gamma$ is blue. Overlaid with the VLA $6 \mathrm{~cm}$ radio contours (Yusef-Zadeh \& Morris 1987a). Numbered stars 8 and 10 , and lettered stars $A$ and $B$ correspond to stars with emission line spectra which were discussed in Paper I.

Fig. 12.- Three color image of G0.18-0.04, the 'Sickle', overlaid with the VLA $6 \mathrm{~cm}$ radio contours (Yusef-Zadeh \& Morris 1987a). $\mathrm{K}^{\prime}$ is red, $\mathrm{H}$ is green, $\mathrm{Br} \gamma$ is blue.

Fig. 13.- Three color image of $\mathrm{G} 0.10+0.02 . \mathrm{K}^{\prime}$ is red, $\mathrm{H}$ is green, $\mathrm{Br} \gamma$ is blue. The source labeled $\mathrm{A}$ in the upper left is a compact source we believe to be an emission line star. The spectrum of this hot young star and a discussion of its stellar type is presented in Paper II.

Fig. 14.- Three color image of the radio peak in the E2 Filament. $\mathrm{K}^{\prime}$ is red, $\mathrm{H}$ is green, 
$\mathrm{Br} \gamma$ is blue.

Fig. 15.- Three color partial image of the compact regions $H 1$, left, and $H 2$, right. $\mathrm{K}^{\prime}$ is red, $\mathrm{H}$ is green, $\mathrm{Br} \gamma$ is blue. See Figure 1 for location relative to the Arched Filaments and the Sgr A West halo. No emission line stars are apparent in this image, but the center of curvature of $\mathrm{H} 1$ was not covered by this scan, so the prescence of a hot star in this region is not precluded.

Fig. 16.- Three color image of the compact region $\mathrm{H} 2 . \mathrm{K}^{\prime}$ is red, $\mathrm{H}$ is green, $\mathrm{Br} \gamma$ is blue. The star labeled $\mathrm{A}$ has an emission spectrum indicative of a $\mathrm{B}[\mathrm{e}]$ star. The star labeled $\mathrm{B}$ has the deep $\mathrm{CO}$ absorption features suggesting an $\mathrm{M}$ supergiant. The stars are discussed in more detail in Paper II.

Fig. 17.- Three color image of the compact regions $\mathrm{H} 5-\mathrm{H} 7 . \mathrm{K}^{\prime}$ is red, $\mathrm{H}$ is green, $\mathrm{Br} \gamma$ is blue. The star located within the emission of $\mathrm{H} 5$, labeled $\mathrm{A}$, shows strong compact $\mathrm{Br} \gamma$ emission and could be a B[e] or possibly an LBV star. See Paper II for details.

Fig. 18. - Three color image of the compact region $\mathrm{H} 8 . \mathrm{K}^{\prime}$ is red, $\mathrm{H}$ is green, $\mathrm{Br} \gamma$ is blue. The star seen in conjunction with the region is the only one to shown $\operatorname{Br} \gamma$ in emissin and is most likely a $B[e]$ star. See Paper II for details.

Fig. 19.- Three color image of the compact regions A-D located to the east of Sgr A East. $\mathrm{K}^{\prime}$ is red, $\mathrm{H}$ is green, $\mathrm{Br} \gamma$ is blue. The emission star marked with the asterisk is a WN 6 star as discussed in Paper II.

Fig. 20.- - Average $\mathrm{H}-\mathrm{K}^{\prime}$ for the QPS region. Each number is centered on the location of the individual image used to derive the color. Each image is $\sim 1^{\prime} \times 1^{\prime}$.

Fig. 21.- Average $\mathrm{H}-\mathrm{K}^{\prime}$ for the E1 \& E2 Filaments. Each number is centered on the location of the individual image used to derive the color. Each image is $\sim 1^{\prime} \times 1^{\prime}$. 
Fig. 22.- Average $\mathrm{H}-\mathrm{K}^{\prime}$ for the $\mathrm{H} 1-\mathrm{H} 8$ region. Each number is centered on the location of the individual image used to derive the color. Each image is $\sim 1^{\prime} \times 1^{\prime}$.

Fig. 23.- Average $\mathrm{H}-\mathrm{K}^{\prime}$ for the Sgr A East region. Each number is centered on the location of the individual image used to derive the color. Each image is $\sim 1^{\prime} \times 1^{\prime}$. 
TABle 1. Observational Limiting Magnitudes

\begin{tabular}{l|c|c|c}
\hline \multicolumn{1}{c|}{ Region } & $\mathrm{J}$ & $\mathrm{H}$ & $\mathrm{K}^{\prime}$ \\
\hline QPS & 17.9 & 16.3 & 14.9 \\
E1 \& E2 Filaments & 18.1 & 15.0 & 13.9 \\
H1-H8 & 18.0 & 16.0 & 14.0 \\
Sgr A East & 17.8 & 15.7 & 14.1 \\
\hline \hline
\end{tabular}


TABle 2A. Median Errors for Continuum Images

\begin{tabular}{|c|c|c|c|c|c|c|c|c|c|}
\hline \multirow[b]{2}{*}{ Case } & \multirow[b]{2}{*}{ Band } & \multicolumn{4}{|c|}{ QPS } & \multicolumn{4}{|c|}{ E1 \& E2 Filaments } \\
\hline & & $\begin{array}{l}\text { Number } \\
\text { of stars }\end{array}$ & Mag & $\sigma$ & DAOPHOT & $\begin{array}{l}\text { Number } \\
\text { of stars }\end{array}$ & Mag & $\sigma$ & DAOPHOT \\
\hline \multirow[t]{2}{*}{$2 \mathrm{~A}$} & $\mathrm{~K}^{\prime}$ & 1659 & 13.46 & 0.076 & 0.065 & \multirow[t]{2}{*}{1636} & 12.00 & 0.050 & 0.034 \\
\hline & $\mathrm{H}$ & & 14.95 & 0.059 & 0.068 & & 13.81 & 0.051 & 0.040 \\
\hline \multirow[t]{2}{*}{$2 \mathrm{~B}$} & $\overline{\mathrm{K}^{\prime}}$ & 515 & 12.18 & 0.072 & 0.040 & \multirow[t]{2}{*}{303} & 10.62 & 0.038 & 0.018 \\
\hline & $\mathrm{J}$ & & 16.62 & 0.052 & 0.061 & & 15.80 & 0.040 & 0.040 \\
\hline \multirow[t]{3}{*}{$2 \mathrm{C}$} & $\overline{\mathrm{K}^{\prime}}$ & 492 & 12.18 & 0.071 & 0.040 & \multirow[t]{3}{*}{299} & 10.61 & 0.040 & 0.018 \\
\hline & $\mathrm{H}$ & & 13.56 & 0.047 & 0.043 & & 12.27 & 0.037 & 0.016 \\
\hline & $\mathrm{J}$ & & 16.65 & 0.052 & 0.062 & & 15.80 & 0.038 & 0.038 \\
\hline \multirow[t]{2}{*}{$4 \mathrm{~A}$} & $\mathrm{~K}^{\prime}$ & 78 & 13.02 & 0.127 & 0.057 & \multirow[t]{2}{*}{103} & 11.81 & 0.078 & $\overline{0.032}$ \\
\hline & $\mathrm{H}$ & & 14.65 & 0.097 & 0.062 & & 13.43 & 0.070 & 0.034 \\
\hline \multirow[t]{2}{*}{$4 \mathrm{~B}$} & $\mathrm{~K}^{\prime}$ & 24 & 11.84 & 0.137 & 0.040 & \multirow[t]{2}{*}{18} & 10.56 & 0.063 & 0.017 \\
\hline & $\mathrm{J}$ & & 16.52 & 0.077 & 0.060 & & 15.50 & 0.065 & 0.030 \\
\hline \multirow[t]{3}{*}{$4 \mathrm{C}$} & $\mathrm{K}^{\prime}$ & 23 & 11.73 & 0.130 & 0.037 & \multirow[t]{3}{*}{17} & 10.05 & 0.061 & 0.017 \\
\hline & $\mathrm{H}$ & & 13.11 & 0.082 & 0.043 & & 11.69 & 0.077 & 0.013 \\
\hline & $\mathrm{J}$ & & 16.47 & 0.077 & 0.059 & & 15.53 & 0.063 & 0.029 \\
\hline
\end{tabular}

TABle 2B. Median Errors for Continuum Images

\begin{tabular}{|c|c|c|c|c|c|c|c|c|c|}
\hline \multirow[b]{2}{*}{ Case } & \multirow[b]{2}{*}{ Band } & \multicolumn{4}{|c|}{$\mathrm{H} 1-\mathrm{H} 8$} & \multicolumn{4}{|c|}{ Sgr A East } \\
\hline & & $\begin{array}{l}\text { Number } \\
\text { of stars }\end{array}$ & Mag & $\sigma$ & DAOPHOT & $\begin{array}{l}\text { Number } \\
\text { of stars }\end{array}$ & Mag & $\sigma$ & DAOPHOT \\
\hline \multirow[t]{2}{*}{$2 \mathrm{~A}$} & $\mathrm{~K}^{\prime}$ & 1289 & 12.87 & 0.046 & 0.037 & \multirow[t]{2}{*}{215} & 12.18 & 0.049 & 0.023 \\
\hline & $\mathrm{H}$ & & 14.65 & 0.056 & 0.046 & & 13.90 & 0.045 & 0.040 \\
\hline \multirow[t]{2}{*}{$2 B$} & $\mathrm{~K}^{\prime}$ & 316 & 11.94 & 0.036 & 0.026 & \multirow[t]{2}{*}{62} & 11.30 & 0.043 & 0.016 \\
\hline & $\mathbf{J}$ & & 16.80 & 0.054 & 0.043 & & 16.00 & 0.039 & 0.030 \\
\hline \multirow[t]{3}{*}{$2 \mathrm{C}$} & $\mathrm{K}^{\prime}$ & 309 & 11.93 & 0.035 & 0.025 & \multirow[t]{3}{*}{62} & 11.30 & 0.043 & 0.016 \\
\hline & $\mathrm{H}$ & & 13.36 & 0.036 & 0.025 & & 12.58 & 0.033 & 0.017 \\
\hline & $\mathrm{J}$ & & 16.79 & 0.052 & 0.042 & & 16.00 & 0.039 & 0.030 \\
\hline \multirow[t]{2}{*}{$\overline{44 \mathrm{~A}}$} & $\overline{\mathrm{K}^{\prime}}$ & 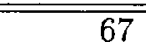 & 12.86 & 0.079 & 0.039 & \multirow[t]{2}{*}{9} & 11.45 & 0.056 & 0.016 \\
\hline & $\mathrm{H}$ & & 14.57 & 0.087 & 0.043 & & 12.91 & 0.064 & 0.030 \\
\hline \multirow[t]{2}{*}{$4 \mathrm{~B}$} & $\mathrm{~K}^{\prime}$ & 13 & 11.70 & 0.071 & 0.030 & \multirow[t]{2}{*}{1} & $\ldots$ & $\ldots$ & $\ldots$ \\
\hline & $\mathrm{J}$ & & 16.59 & 0.075 & 0.043 & & $\ldots$ & $\ldots$ & $\ldots$ \\
\hline \multirow[t]{3}{*}{$4 \mathrm{C}$} & $\mathrm{K}^{\prime}$ & 13 & 11.71 & 0.072 & 0.030 & \multirow[t]{3}{*}{1} & $\ldots$ & $\ldots$ & .. \\
\hline & $\mathrm{H}$ & & 13.36 & 0.057 & 0.027 & & $\ldots$ & $\ldots$ & $\ldots$ \\
\hline & $J$ & & 16.58 & 0.075 & 0.040 & & $\ldots$ & $\ldots$ & $\ldots$ \\
\hline
\end{tabular}


TABle 3. Intrinsic Infrared Magnitudes

\begin{tabular}{|c|c|c|c|c|c|}
\hline Type & $\mathrm{MV}_{\mathrm{V}}^{\mathrm{a}}$ & $\mathrm{M}_{\mathrm{K}}$ & $\mathrm{M}_{\mathrm{K}^{\prime}}{ }^{\mathrm{b}}$ & $\mathrm{M}_{\mathrm{H}}$ & $\mathrm{M}_{\boldsymbol{J}}$ \\
\hline & \multicolumn{5}{|c|}{ Main Sequence (V) } \\
\hline$O 5^{\mathrm{c}}$ & -5.7 & -4.81 & -4.82 & -4.89 & -4.97 \\
\hline $\mathrm{B} 0^{\mathrm{c}}$ & -4.1 & -3.34 & -3.35 & -3.41 & -3.52 \\
\hline $\mathrm{B}^{\mathrm{c}}$ & -1.1 & -0.68 & -0.70 & -0.77 & -0.77 \\
\hline$A 0^{d}$ & 0.7 & 0.70 & 0.70 & 0.70 & 0.70 \\
\hline $\mathrm{F}^{\mathrm{d}}$ & 2.6 & 1.90 & 1.91 & 1.93 & 2.06 \\
\hline $\mathrm{GO}^{\mathrm{d}}$ & 4.4 & 2.99 & 3.0 & 3.04 & 3.35 \\
\hline $\mathrm{K} 0^{\mathrm{d}}$ & 5.9 & 3.94 & 3.95 & 4.02 & 4.47 \\
\hline \multirow[t]{2}{*}{$M 0^{d}$} & 9.0 & 5.35 & 5.37 & 5.52 & 6.21 \\
\hline & \multicolumn{3}{|c|}{ Giants (III) } & & \\
\hline$O 5^{c}$ & -5.4 & -4.52 & -4.53 & -4.60 & -4.72 \\
\hline$B 5^{c}$ & -5.0 & -4.31 & -4.33 & -4.40 & -4.49 \\
\hline $\mathrm{B} 0^{\mathrm{c}}$ & -2.4 & -1.99 & -2.00 & -2.05 & -2.09 \\
\hline $\mathrm{GO}^{\mathrm{d}}$ & 1.1 & -0.65 & -0.64 & -0.59 & -0.20 \\
\hline $\mathrm{G} 5^{\mathrm{d}}$ & 0.7 & -1.40 & -1.39 & -1.32 & -0.84 \\
\hline$K 0^{d}$ & 0.5 & -1.81 & -1.79 & -1.72 & -1.18 \\
\hline$K 5^{d}$ & -0.2 & -3.80 & -3.77 & -3.64 & -2.85 \\
\hline $\mathrm{M} 0^{\mathrm{d}}$ & -0.4 & -4.25 & -4.22 & -4.06 & -3.24 \\
\hline \multirow[t]{2}{*}{$\mathrm{M}^{\mathrm{d}}$} & -0.8 & -6.76 & -6.71 & -6.48 & -5.53 \\
\hline & \multicolumn{3}{|c|}{ Supergiants (Ia) } & & \\
\hline $\mathrm{O} 5^{\mathrm{c}}$ & -8.4 & -7.50 & -7.51 & -7.53 & -7.69 \\
\hline $\mathrm{B}^{\mathrm{c}}$ & -6.7 & -6.09 & -6.09 & -6.11 & -6.22 \\
\hline$B 5^{\mathrm{c}}$ & -6.9 & -6.69 & -6.69 & -6.69 & -6.76 \\
\hline $\mathrm{K} 5(\mathrm{Ib})^{\mathrm{e}}$ & -4.6 & -8.10 & 8.08 & -7.96 & -7.35 \\
\hline $\mathrm{MO}^{\mathrm{e}}$ & -7.5 & -11.3 & -11.3 & -11.13 & -10.58 \\
\hline $\mathrm{M} 2^{\mathrm{e}}$ & -7 & -11.1 & -11.1 & -10.89 & -10.34 \\
\hline
\end{tabular}

aAllen, Astrophysical Quantities

bDerived from equation (1)

'Infrared colors from Wegner \& Walter (1994)

dInfrared colors from Bessel \& Brett (1988)

e Infrared colors from Elias et al. (1985) 
Table 4. H-K', J-H, and Average Number of stars by Location and Stellar Type

\begin{tabular}{|c|c|c|c|c|c|c|}
\hline & \multicolumn{2}{|c|}{$\mathrm{H}-\mathrm{K}^{\prime}$} & \multicolumn{2}{|c|}{$\mathrm{J}-\mathrm{H}$} & \multicolumn{2}{|c|}{ Ave. \# of stars/Frame } \\
\hline & OB & KM & OB & $\mathrm{KM}$ & $\mathrm{OB}$ & $\mathrm{KM}$ \\
\hline \multicolumn{7}{|c|}{ QPS } \\
\hline On & $1.59 \pm 0.01$ & $1.48 \pm 0.01$ & $3.26 \pm 0.01$ & $3.36 \pm 0.01$ & 20 & 32 \\
\hline Off & $1.60 \pm 0.02$ & $1.49 \pm 0.01$ & $3.27 \pm 0.05$ & $3.50 \pm 0.01$ & 21 & 20 \\
\hline Straight & $1.55 \pm 0.02$ & $1.45 \pm 0.01$ & $3.19 \pm 0.04$ & $3.37 \pm 0.03$ & 11 & 30 \\
\hline Row 1 & $1.53 \pm 0.01$ & $1.45 \pm 0.01$ & $3.28 \pm 0.03$ & $3.38 \pm 0.02$ & 14 & 22 \\
\hline Row 2 & $1.60 \pm 0.01$ & $1.52 \pm 0.01$ & $3.34 \pm 0.03$ & $3.55 \pm 0.01$ & 23 & 29 \\
\hline Row 3 & $1.58 \pm 0.01$ & $1.45 \pm 0.01$ & $3.31 \pm 0.02$ & $3.38 \pm 0.02$ & 26 & 30 \\
\hline \multicolumn{7}{|c|}{ E1 and E2 Filaments } \\
\hline On & $1.89 \pm 0.04$ & $1.73 \pm 0.04$ & $3.91 \pm 0.10$ & $3.88 \pm 0.07$ & 5 & 10 \\
\hline Off & $1.96 \pm 0.09$ & $1.80 \pm 0.03$ & $4.03 \pm 0.19$ & $4.03 \pm 0.05$ & 6 & 12 \\
\hline Straight & $1.67 \pm 0.01$ & $1.56 \pm 0.01$ & $3.46 \pm 0.01$ & $3.59 \pm 0.01$ & 8 & 15 \\
\hline Row 1 & $1.66 \pm 0.01$ & $1.54 \pm 0.02$ & $3.44 \pm 0.01$ & $3.58 \pm 0.01$ & 8 & 16 \\
\hline Row 2 & $1.85 \pm 0.04$ & $1.74 \pm 0.04$ & $3.90 \pm 0.14$ & $3.92 \pm 0.08$ & 9 & 13 \\
\hline Row 3 & $1.80 \pm 0.02$ & $1.78 \pm 0.03$ & $3.75 \pm 0.01$ & $3.97 \pm 0.04$ & 4 & 12 \\
\hline Row 4 & $1.98 \pm 0.10$ & $1.81 \pm 0.10$ & $4.12 \pm 0.23$ & $4.09 \pm 0.22$ & 5 & 7 \\
\hline \multicolumn{7}{|c|}{$\mathrm{H} 1-\mathrm{H} 8$} \\
\hline On & $1.74 \pm 0.02$ & $1.58 \pm 0.01$ & $3.59 \pm 0.02$ & $3.65 \pm 0.03$ & 7 & 15 \\
\hline NW & $1.83 \pm 0.02$ & $1.60 \pm 0.02$ & $3.75 \pm 0.05$ & $3.58 \pm 0.02$ & 10 & 7 \\
\hline $\mathrm{SE}$ & $1.63 \pm 0.05$ & $1.56 \pm 0.01$ & $3.44 \pm 0.03$ & $3.60 \pm 0.01$ & 8 & 20 \\
\hline Row 1 & $1.86 \pm 0.02$ & $1.63 \pm 0.01$ & $3.78 \pm 0.02$ & $3.69 \pm 0.02$ & 6 & 11 \\
\hline Row 2 & $1.77 \pm 0.03$ & $1.64 \pm 0.02$ & $3.64 \pm 0.07$ & $3.77 \pm 0.07$ & 8 & 14 \\
\hline Row 3 & $1.65 \pm 0.04$ & $1.53 \pm 0.02$ & $3.44 \pm 0.05$ & $3.54 \pm 0.01$ & 9 & 22 \\
\hline \multicolumn{7}{|c|}{ Sgr A East } \\
\hline Column 1 & $1.51 \pm 0.03$ & $1.69 \pm 0.01$ & $3.33 \pm 0.15$ & $3.93 \pm 0.04$ & 1 & 14 \\
\hline Column 2 & $1.59 \pm 0.03$ & $1.45 \pm 0.01$ & $3.39 \pm 0.06$ & $3.39 \pm 0.02$ & 7 & 12 \\
\hline Column 3 & $1.85 \pm 0.31$ & $1.48 \pm 0.02$ & $3.97 \pm 0.68$ & $3.51 \pm 0.05$ & 1 & 10 \\
\hline
\end{tabular}


TABle 5. Observed vs. Theoretical $\operatorname{Br} \gamma$ emission

\begin{tabular}{|c|c|c|c|c|}
\hline Region & Figure & $\begin{array}{c}\text { Measured } \mathrm{Br} \gamma \\
\left(10^{-20} \mathrm{~W} \mathrm{~cm}^{-2}\right)\end{array}$ & $\begin{array}{l}6 \mathrm{~cm} \\
(\mathrm{mJy})\end{array}$ & $r_{B r \gamma}$ \\
\hline \multicolumn{5}{|c|}{ QPS } \\
\hline Pistol & 13 & $5.73 \pm 0.08$ & 520 & 2.6 \\
\hline Sickle & 14 & $12.6 \pm 1.2$ & 1000 & 2.4 \\
\hline \multicolumn{5}{|c|}{ E1 \& E2 Filaments } \\
\hline $\mathrm{G} 0.10+0.02$ & 11 & $19.0 \pm 0.7$ & 990 & 2.0 \\
\hline E2 Peak & 12 & $19.5 \pm 3.0$ & 260 & 0.6 \\
\hline \multicolumn{5}{|c|}{$\mathrm{H} 1-\mathrm{H} 8$} \\
\hline $\mathrm{H} 1$ & 15 & $2.2 \pm 0.1$ & 470 & 3.4 \\
\hline $\mathrm{H} 2$ & 16 & $11.7 \pm 4$ & 500 & 1.8 \\
\hline H5(W) & 17 & $6.2 \pm 0.4$ & 510 & 2.5 \\
\hline $\mathrm{H} 5(\mathrm{E})$ & 17 & $0.63 \pm 0.08$ & 45 & 2.3 \\
\hline $\mathrm{H} 6$ & 17 & $0.076 \pm 0.003$ & 16 & 3.4 \\
\hline $\mathrm{H} 7$ & 17 & $0.39 \pm 0.10$ & 45 & 2.8 \\
\hline $\mathrm{H} 8$ & 18 & $0.45 \pm 0.01$ & 16 & 1.6 \\
\hline \multicolumn{5}{|c|}{ Sgr A East } \\
\hline A & 19 & $17.1 \pm 0.4$ & 420 & 1.2 \\
\hline B & 19 & $3.2 \pm 0.2$ & 100 & 1.5 \\
\hline $\mathrm{C} 1$ & 19 & $3.1 \pm 0.2$ & 87 & 1.4 \\
\hline $\mathrm{C} 2$ & 19 & $1.1 \pm 0.1$ & 24 & 1.2 \\
\hline $\mathrm{D}$ & 19 & $0.63 \pm 0.06$ & 42 & 2.3 \\
\hline
\end{tabular}


TABLE 6. Comparison of $A_{K}$

\begin{tabular}{|c|c|c|c|}
\hline & Photometry & $\begin{array}{l}\text { Derived from } \\
\text { Br } \gamma \text { Extinction }\end{array}$ & $\begin{array}{c}\text { Derived from } \mathrm{Br} \gamma \\
\text { (assuming radio losses) }\end{array}$ \\
\hline Pistol & 3.0 & 2.7 & 3.0 \\
\hline Sickle & 3.0 & 2.5 & 3.2 \\
\hline $\mathrm{G} 0.10+0.02$ & 4.2 & 2.1 & 2.8 \\
\hline E2 Peak & 3.3 & 0.6 & 1.4 \\
\hline $\mathrm{H} 1$ & 3.6 & 3.6 & 4.3 \\
\hline $\mathrm{H} 2$ & 3.7 & 1.9 & 2.6 \\
\hline $\mathrm{H} 5$ & 3.6 & 2.5 & 3.2 \\
\hline $\mathrm{H} 6$ & 3.6 & 3.6 & 4.3 \\
\hline $\mathrm{H} 7$ & 3.6 & 2.9 & 3.6 \\
\hline $\mathrm{H} 8$ & 3.6 & 1.7 & 2.4 \\
\hline $\mathrm{A}$ & 2.8 & 1.3 & 2.1 \\
\hline B & 2.8 & 1.6 & 2.3 \\
\hline $\mathrm{C} 1$ & 2.8 & 1.5 & 2.2 \\
\hline C2 & 2.8 & 1.3 & 1.9 \\
\hline $\mathrm{D}$ & 2.8 & 2.4 & 3.1 \\
\hline
\end{tabular}




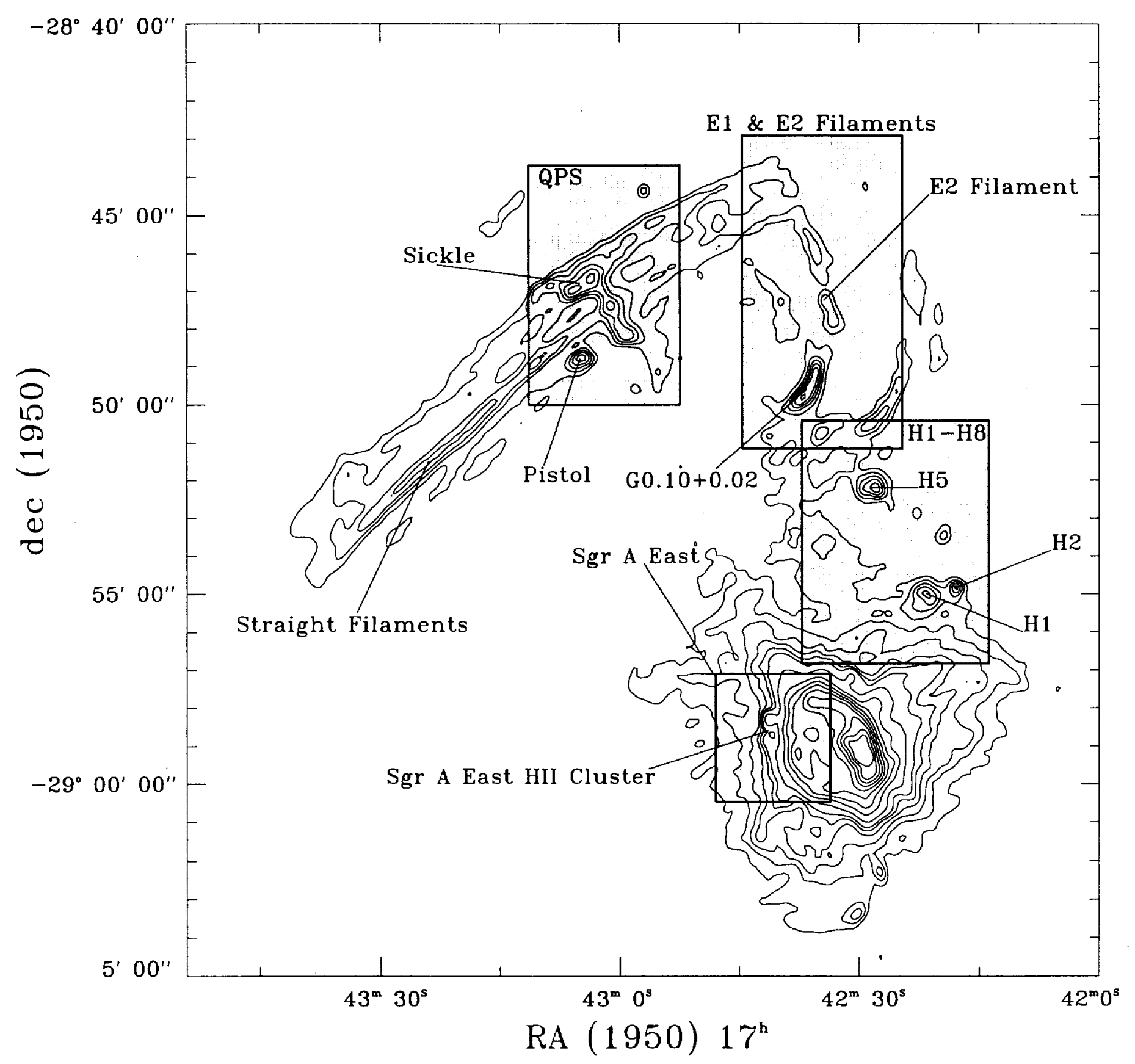




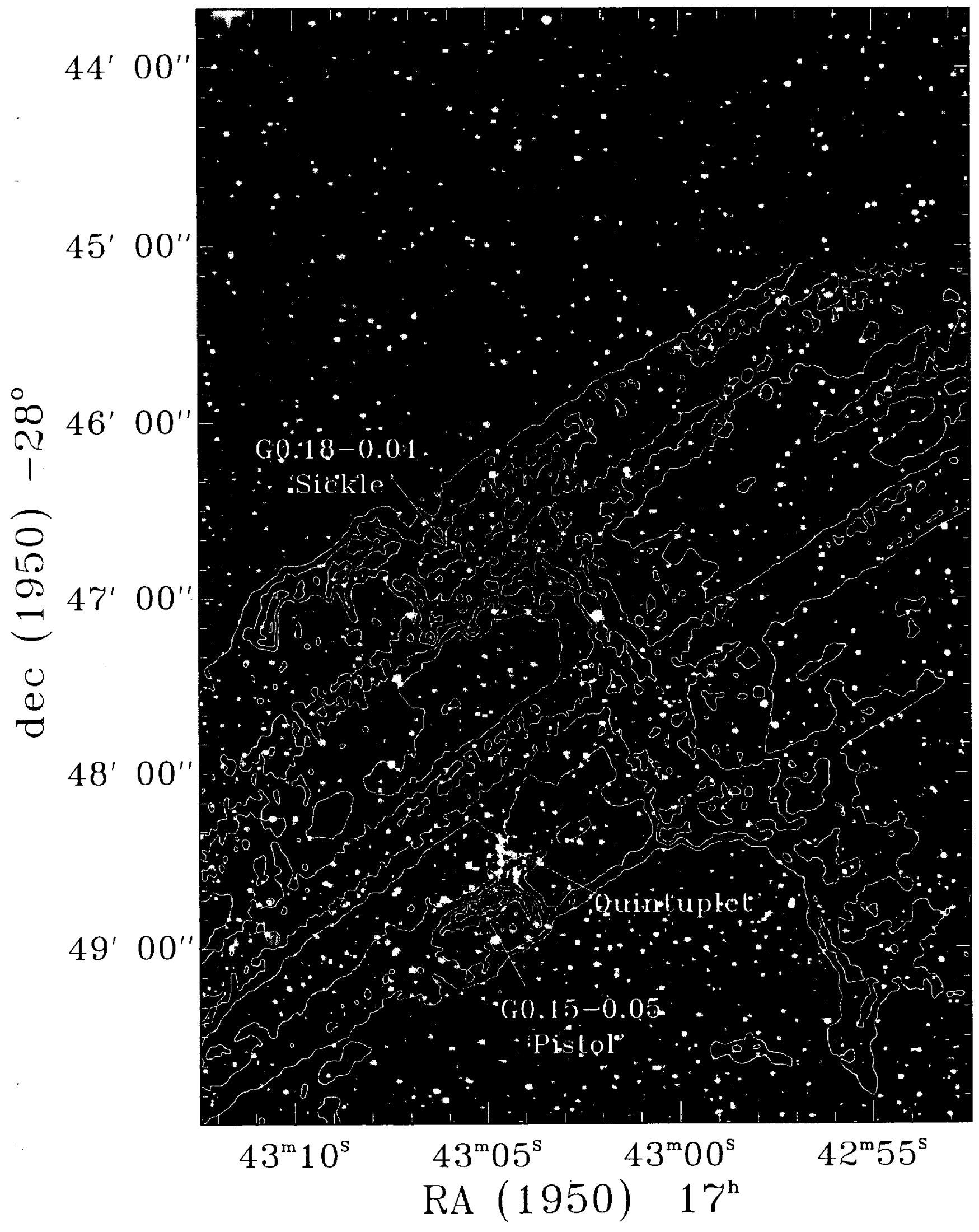




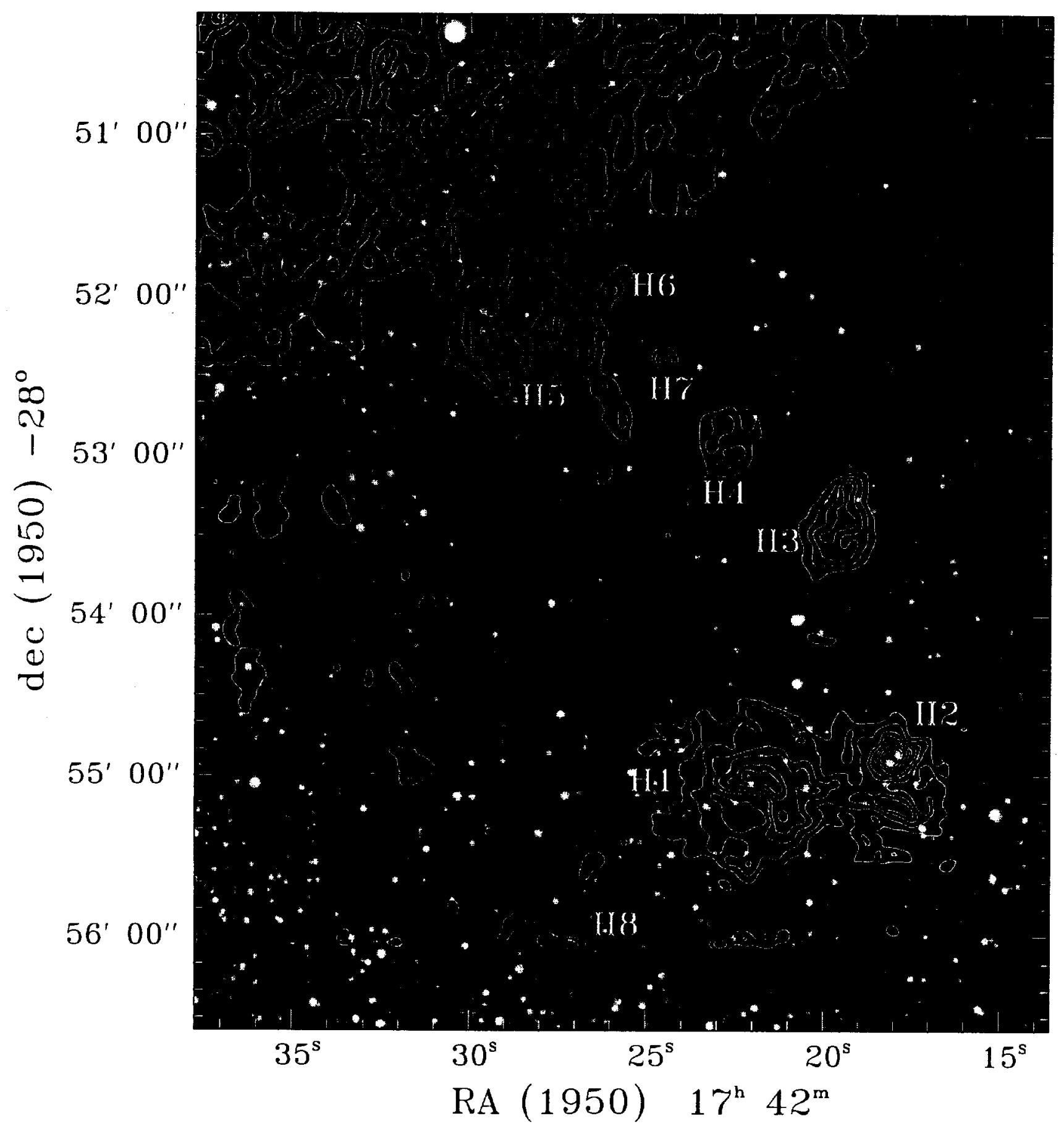




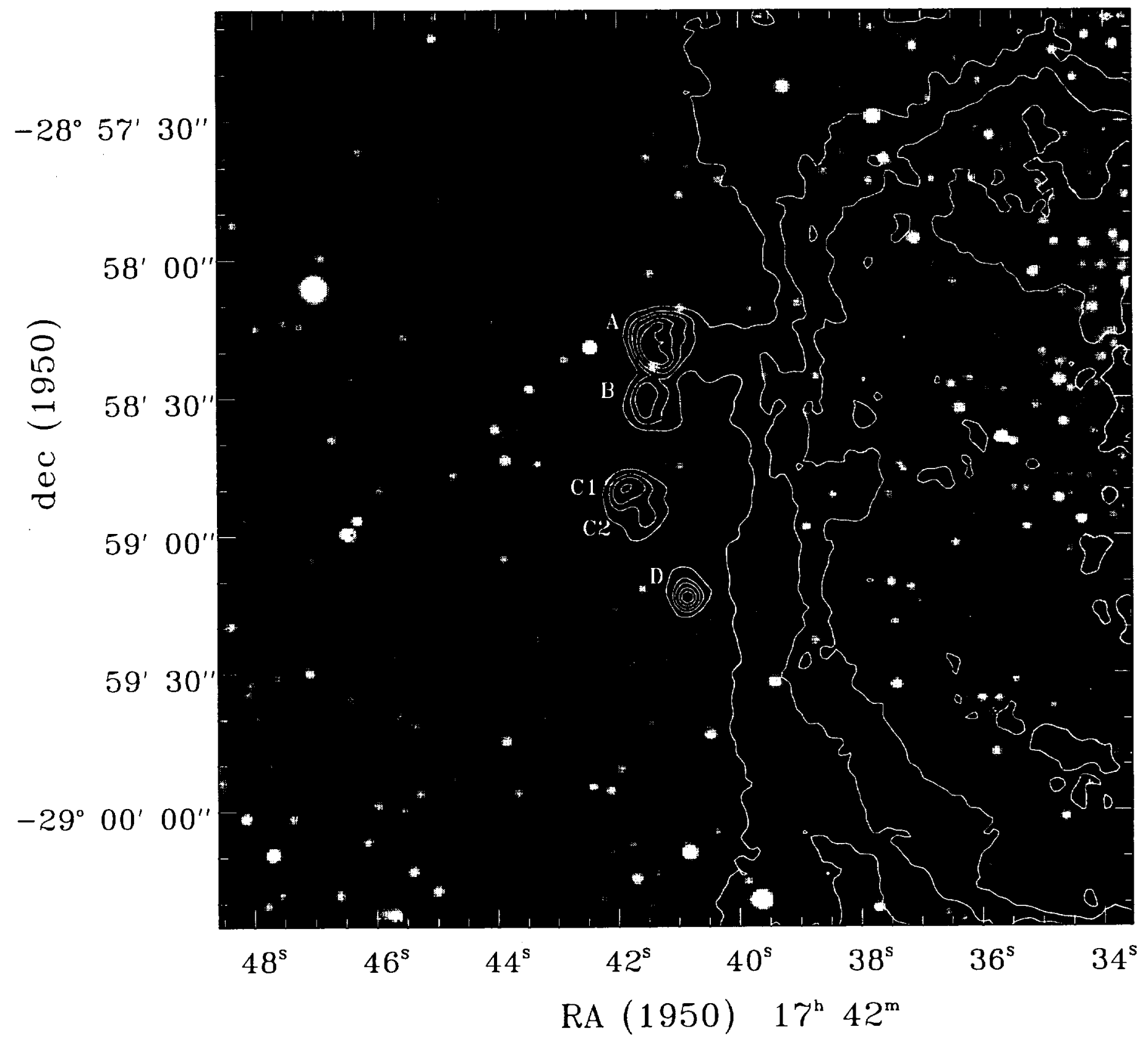



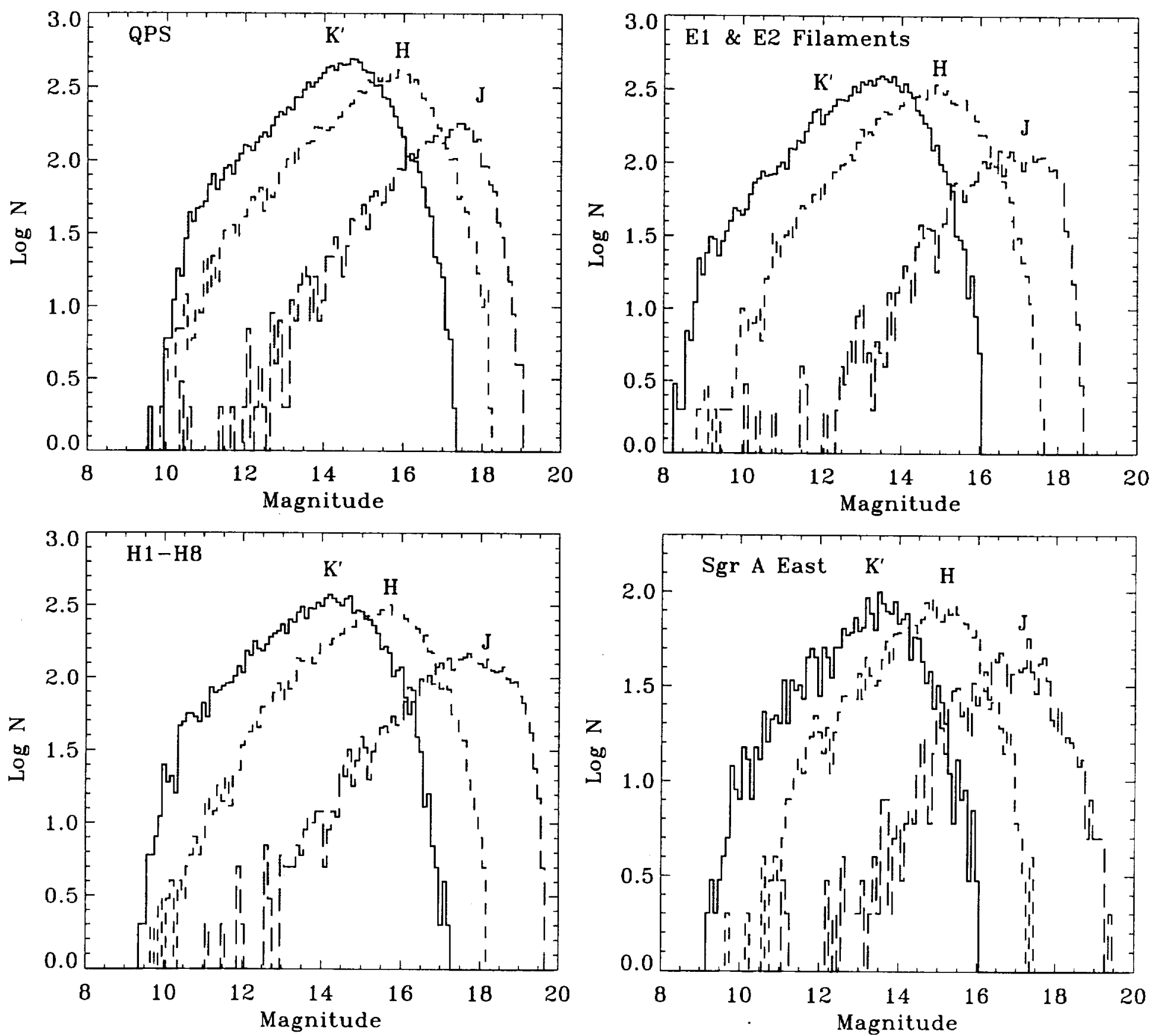

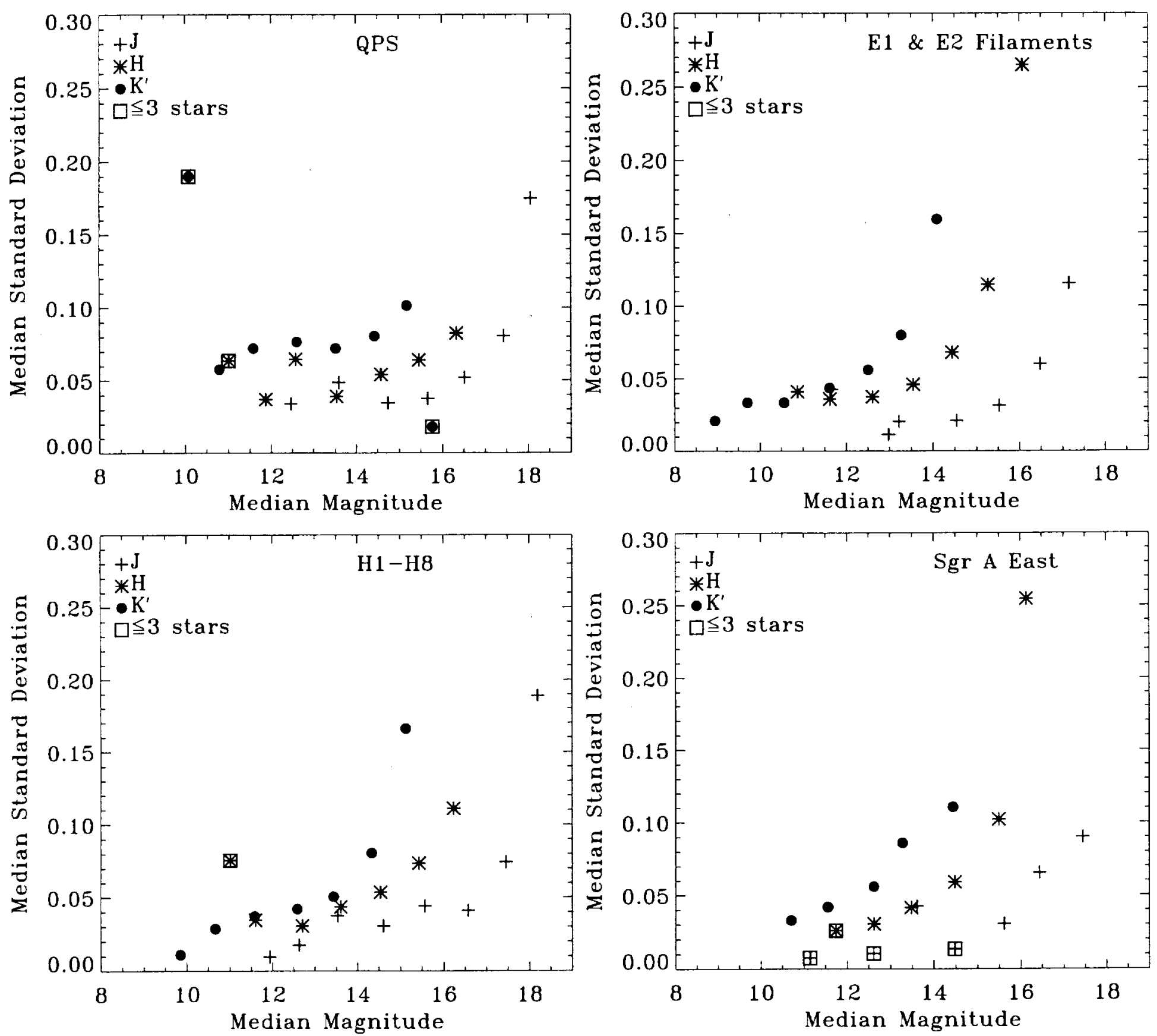

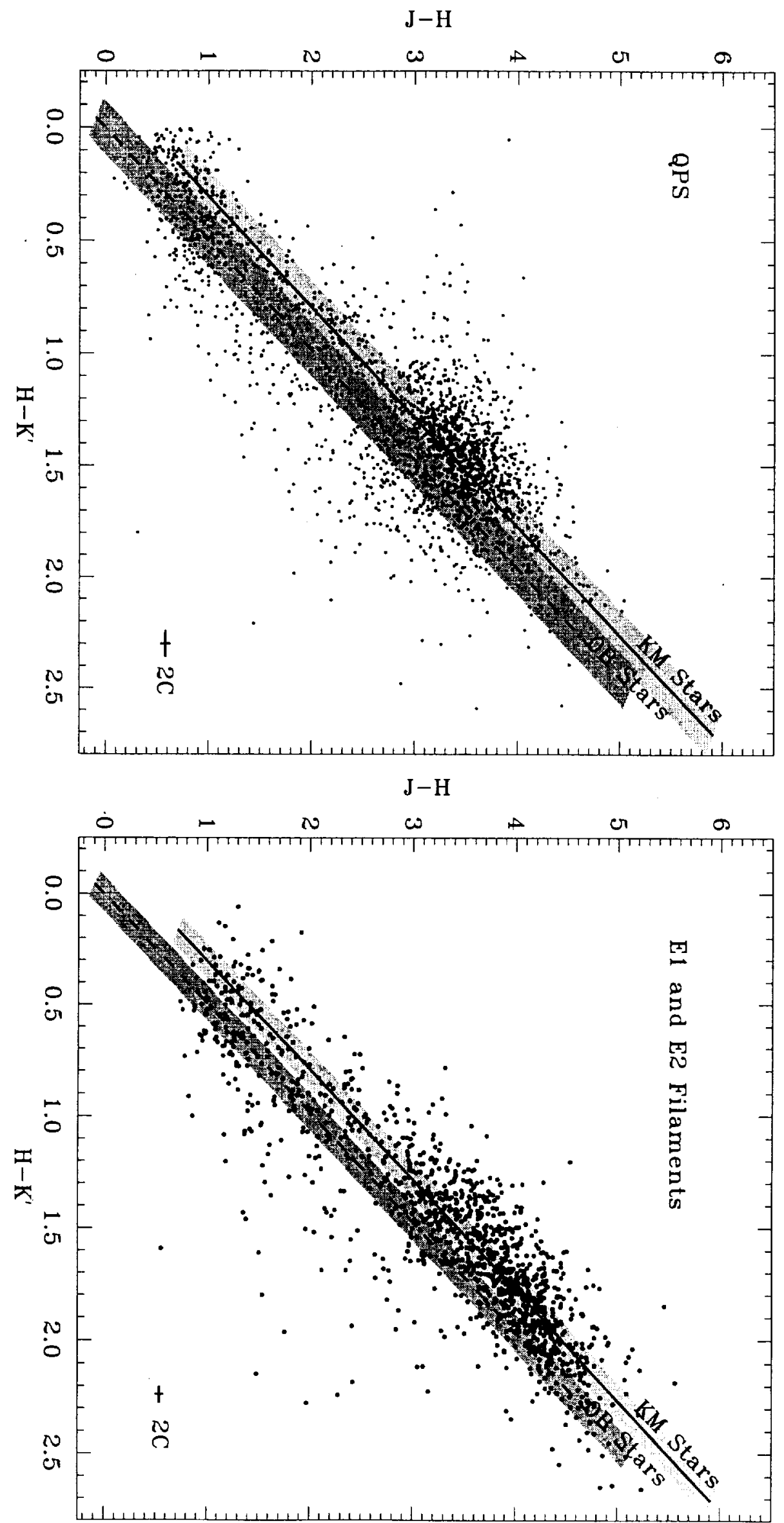

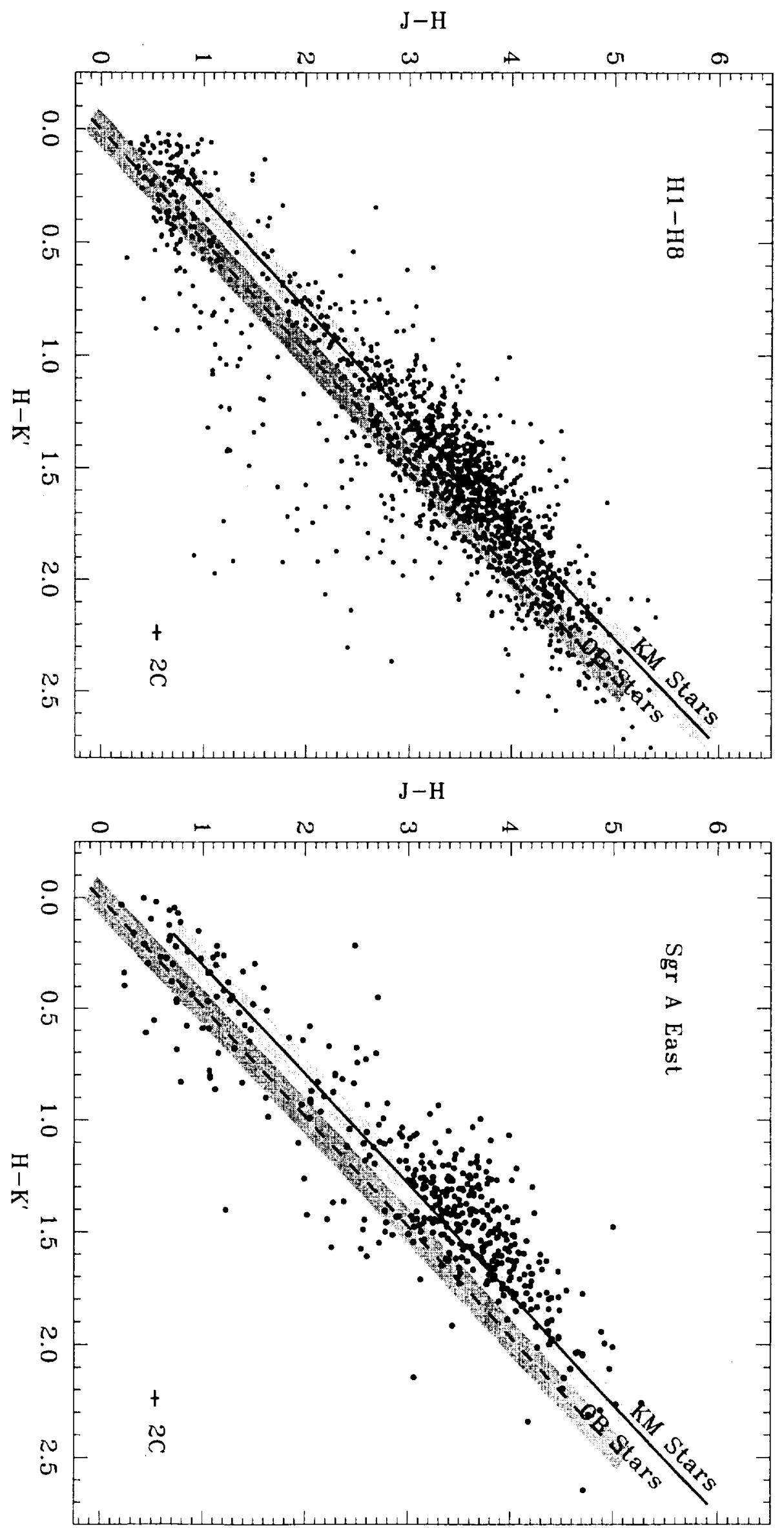

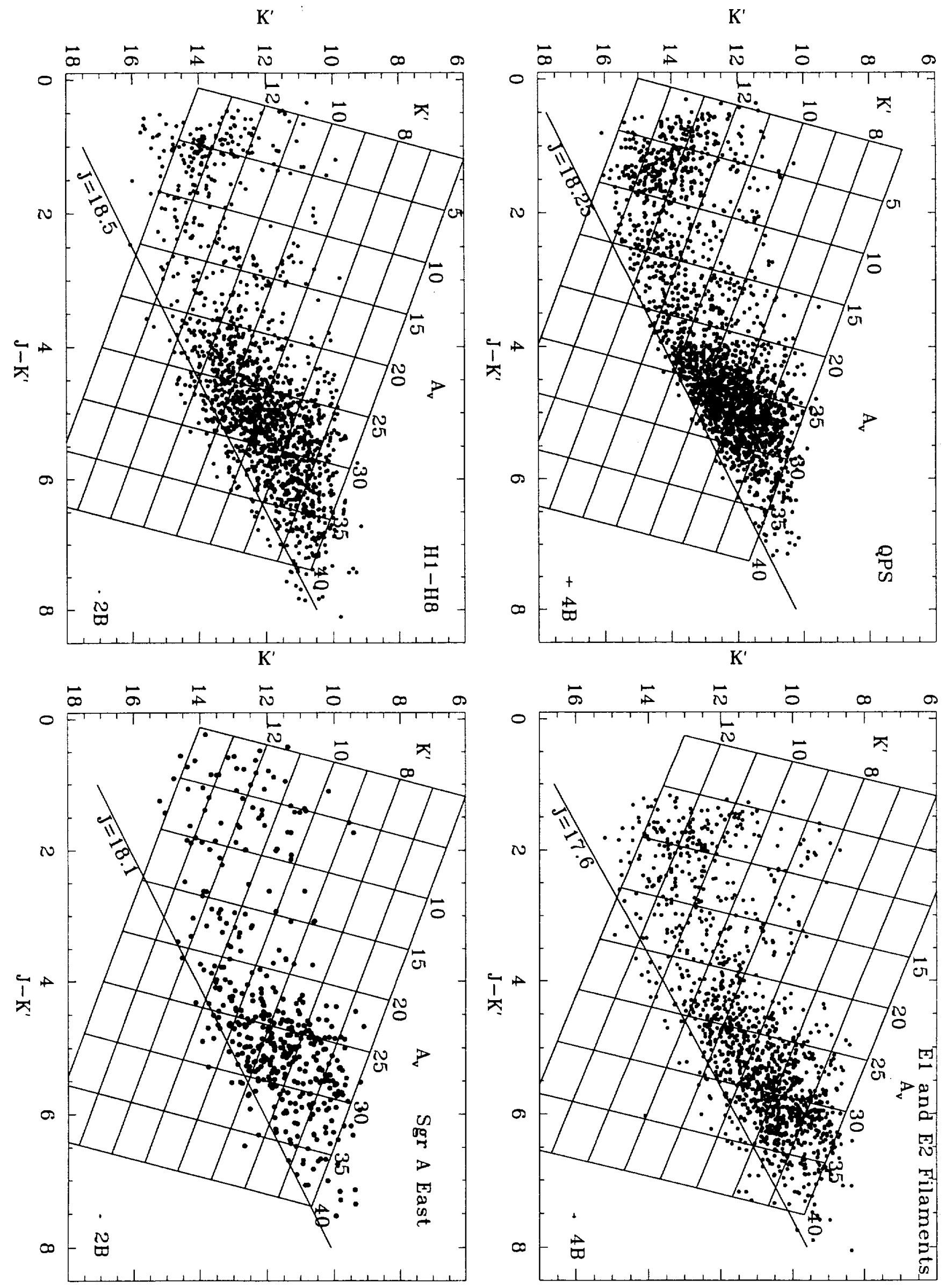

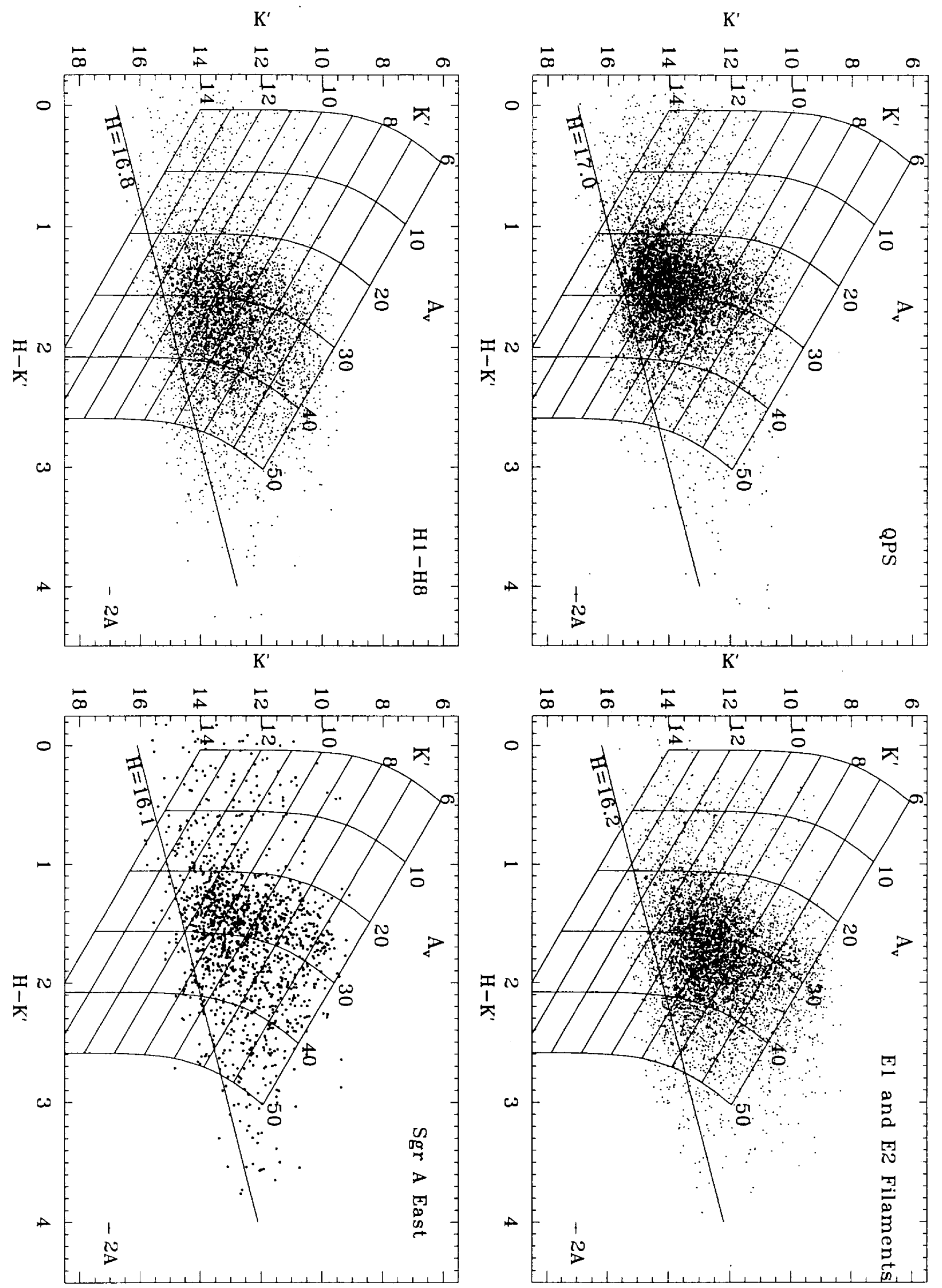


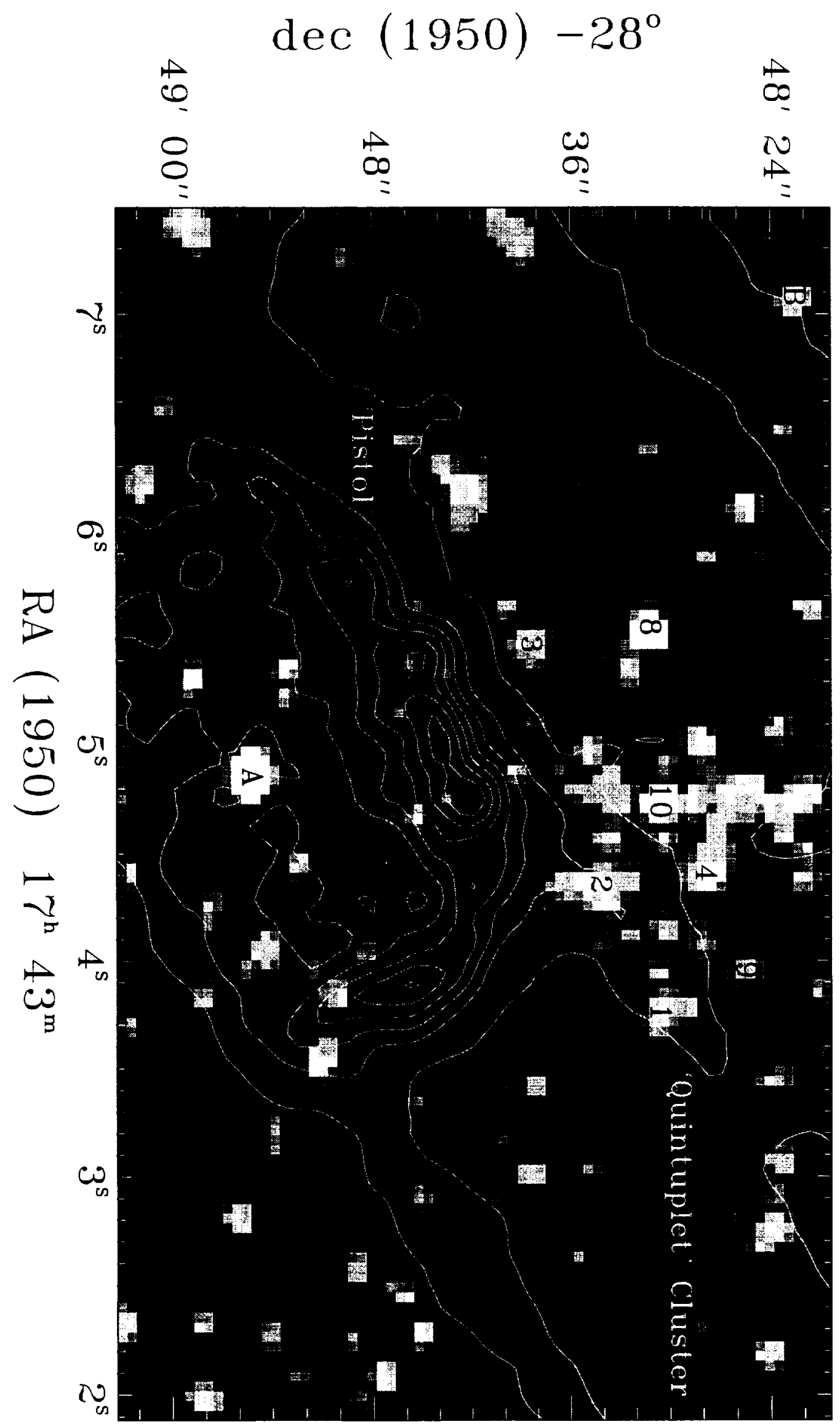




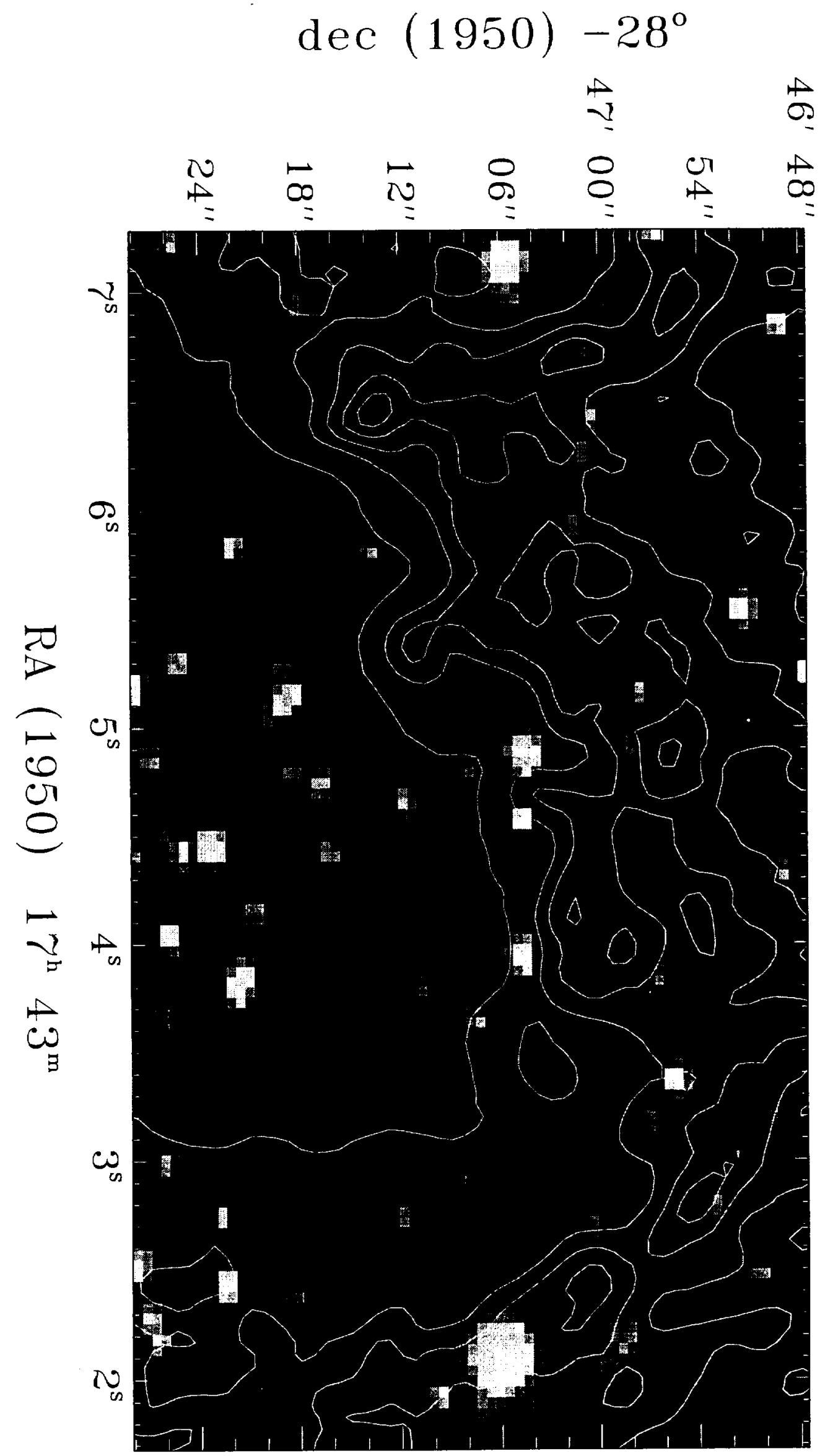




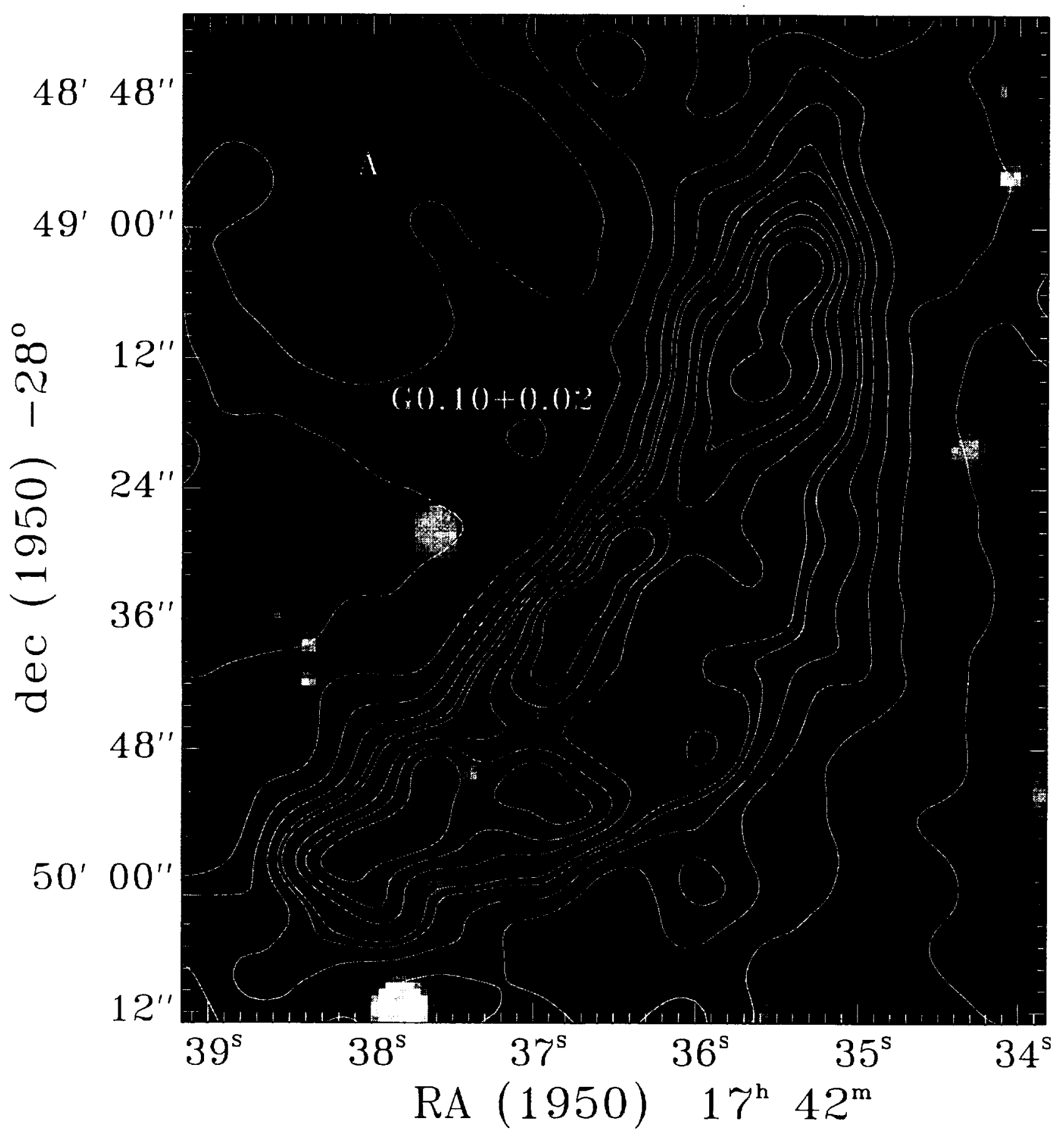




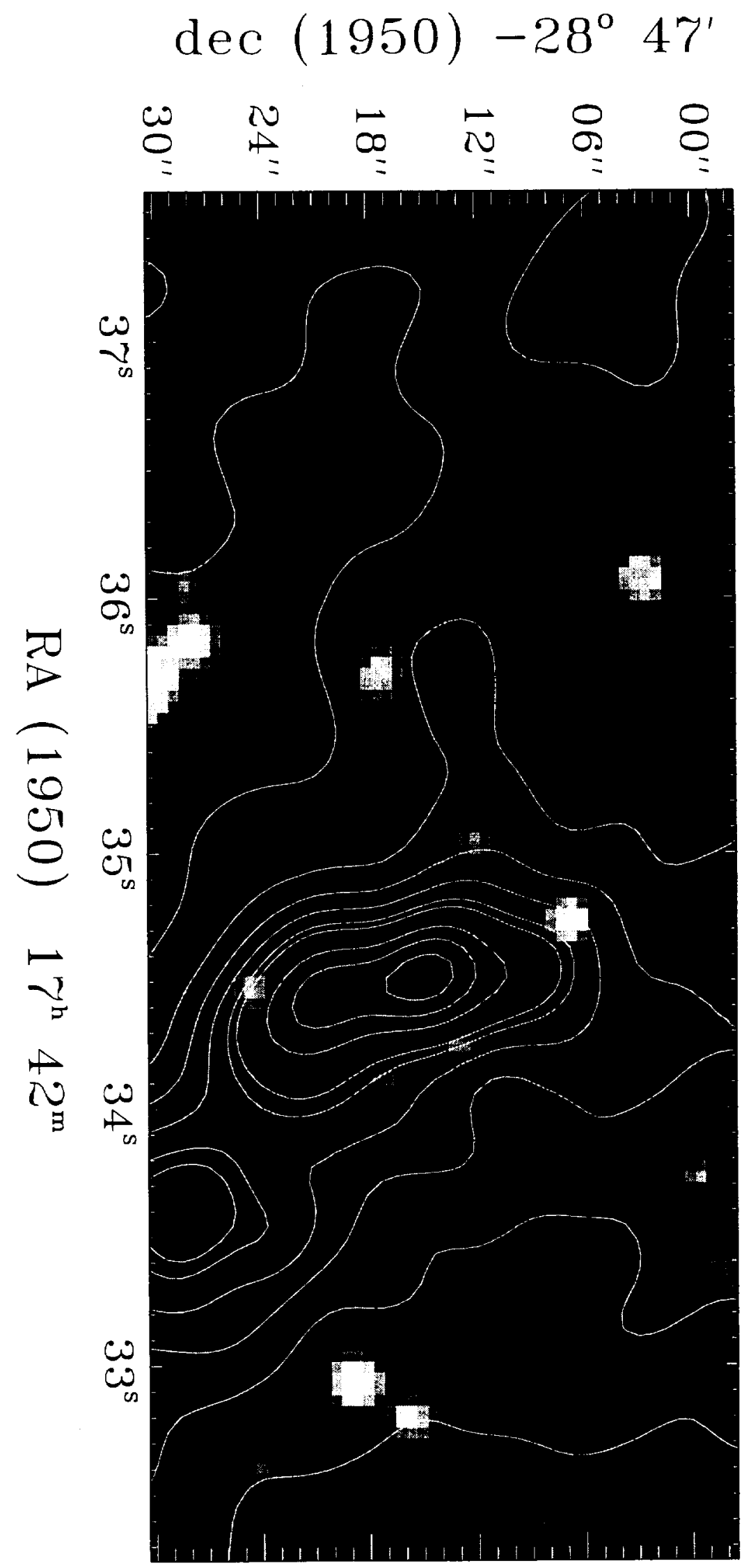




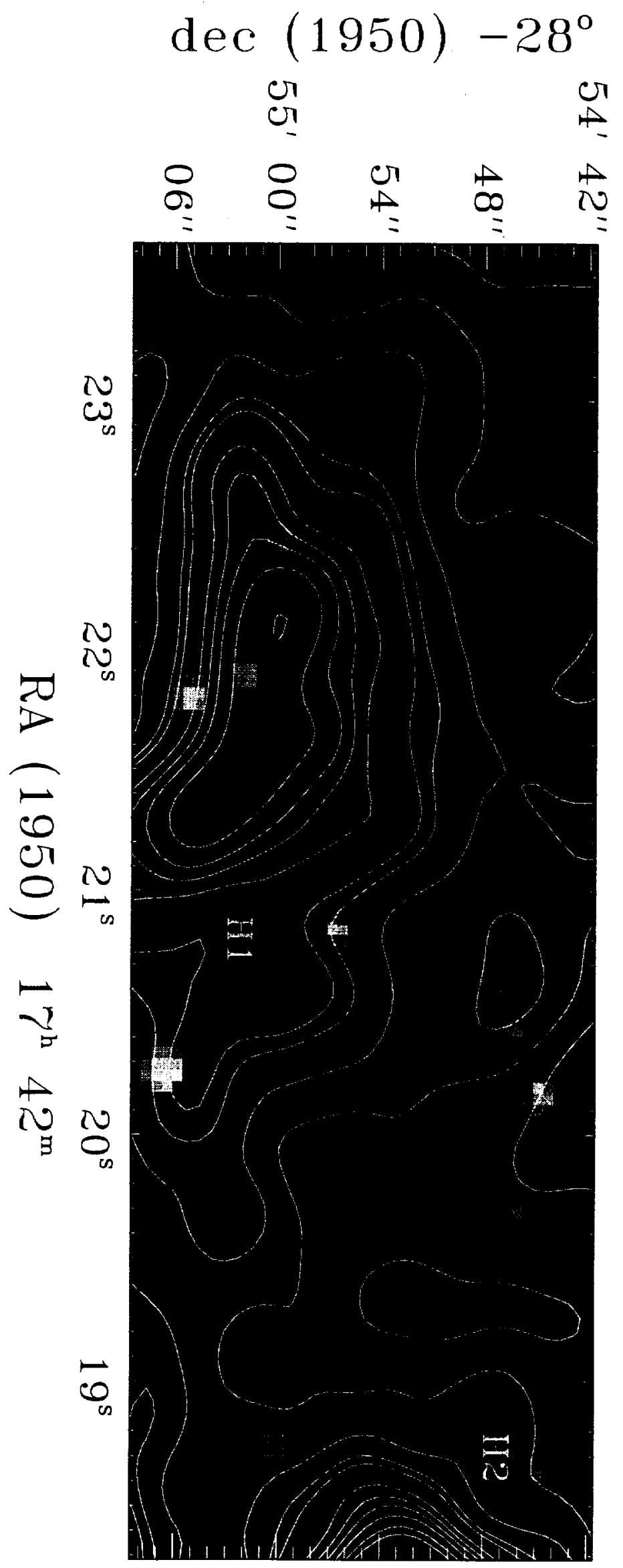




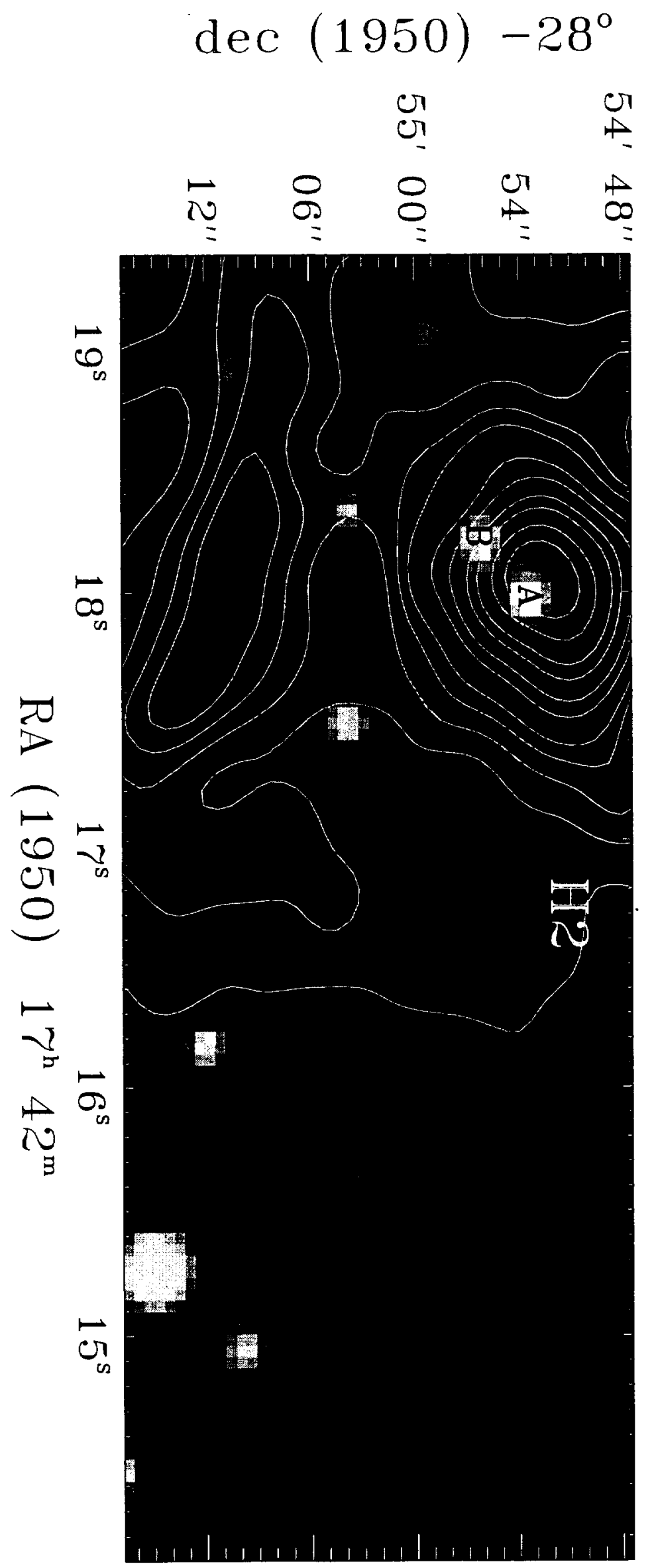




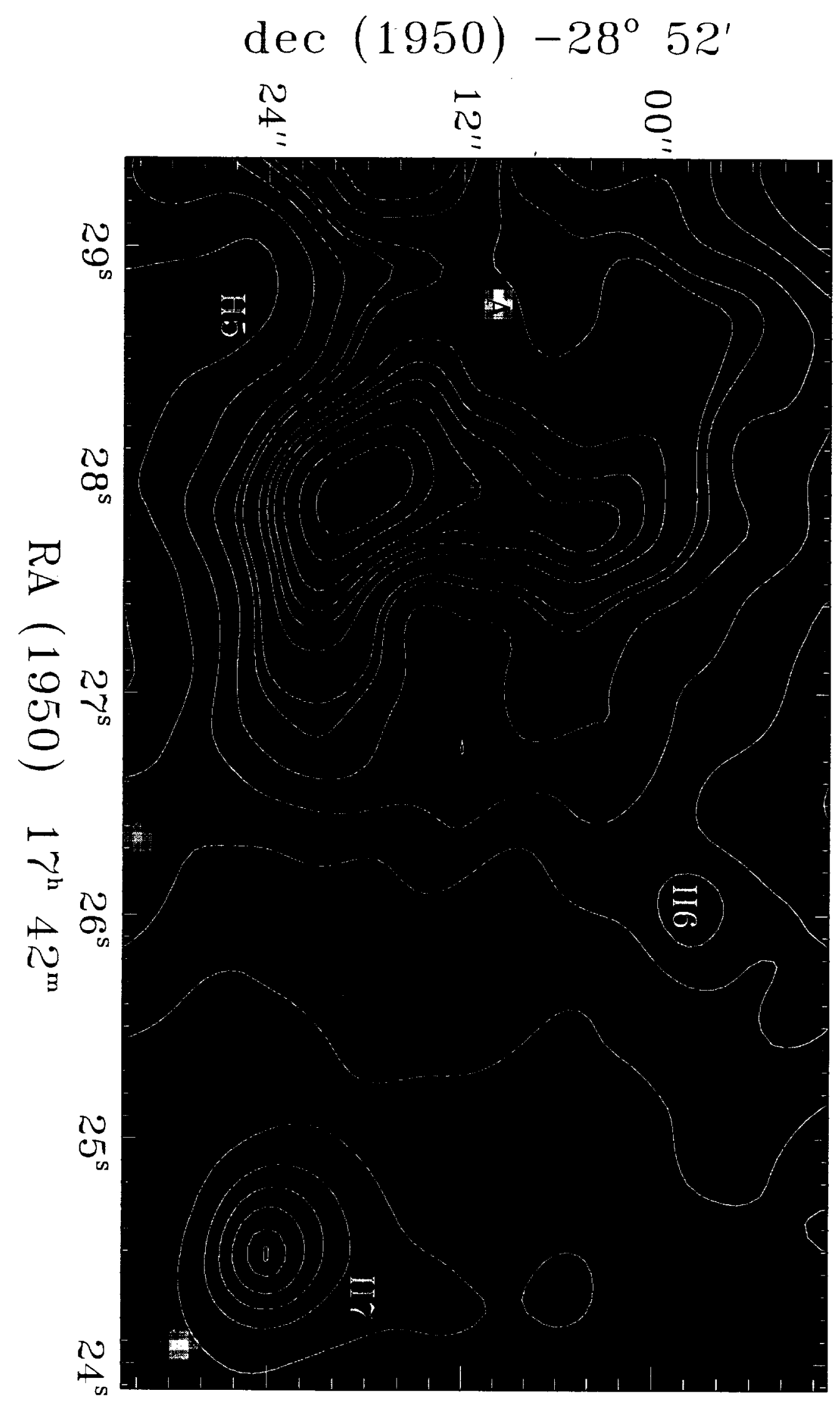




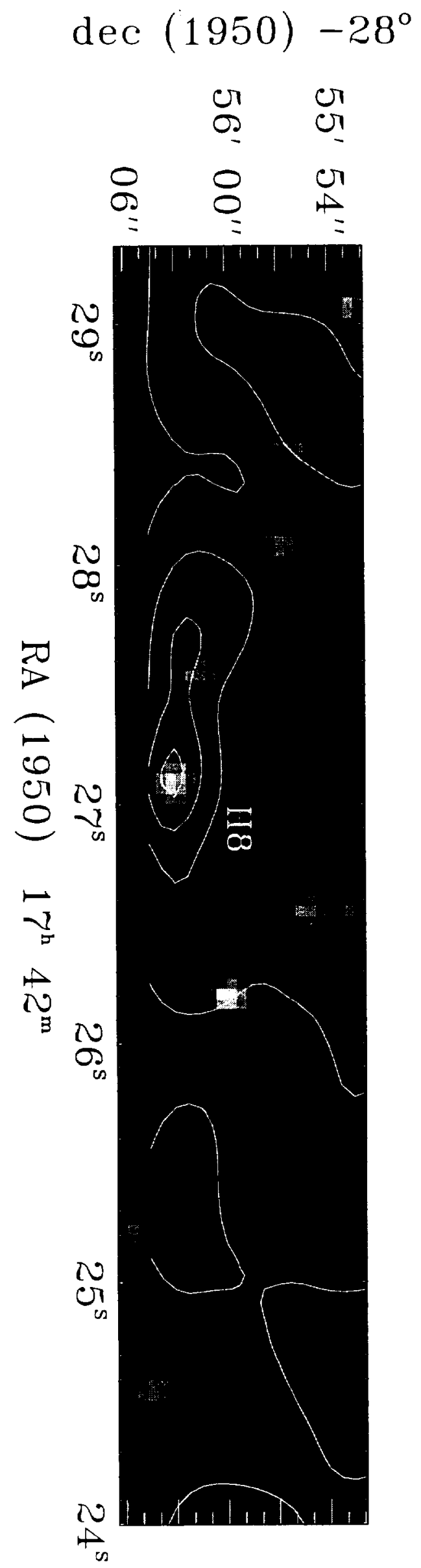




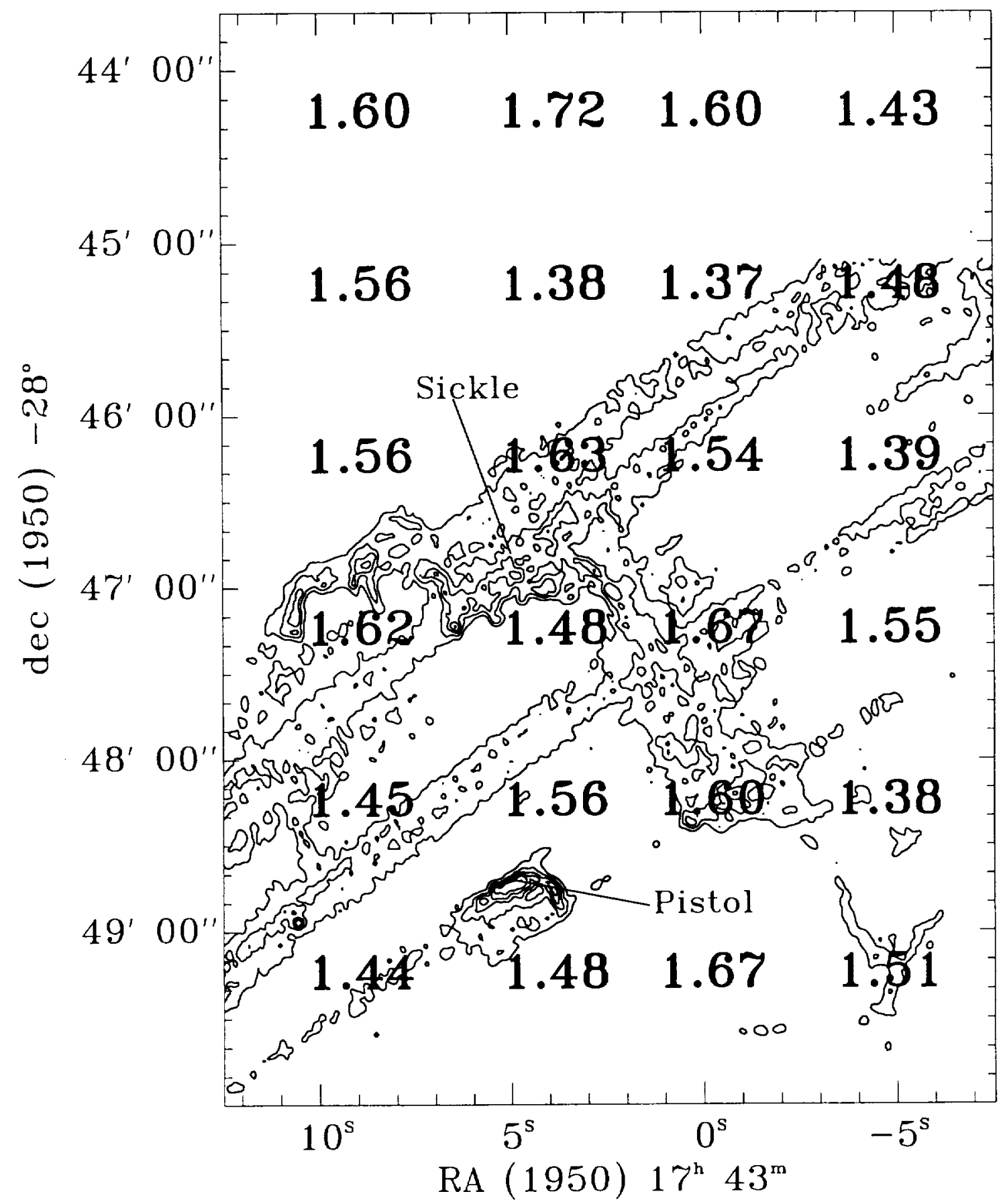




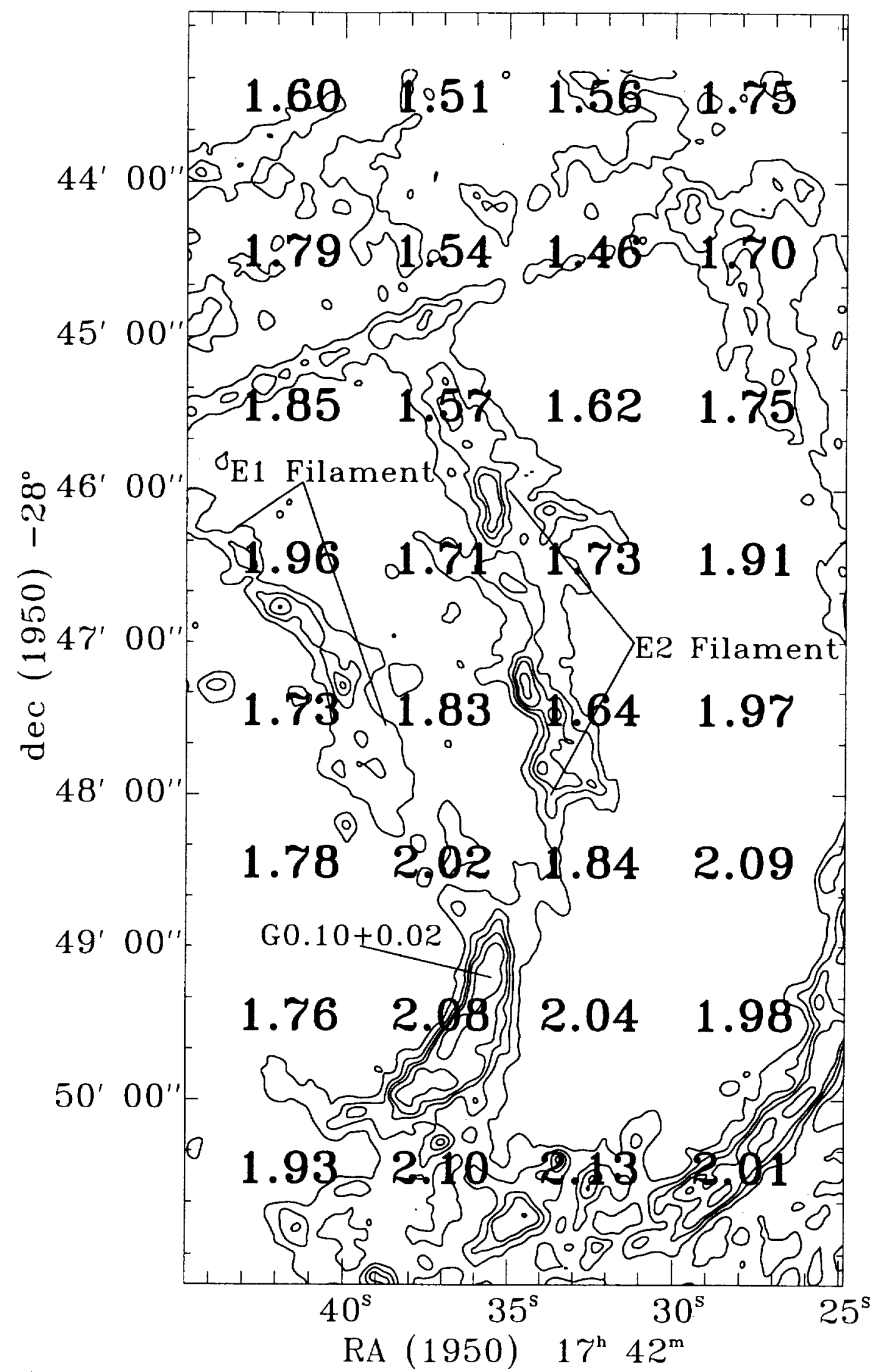




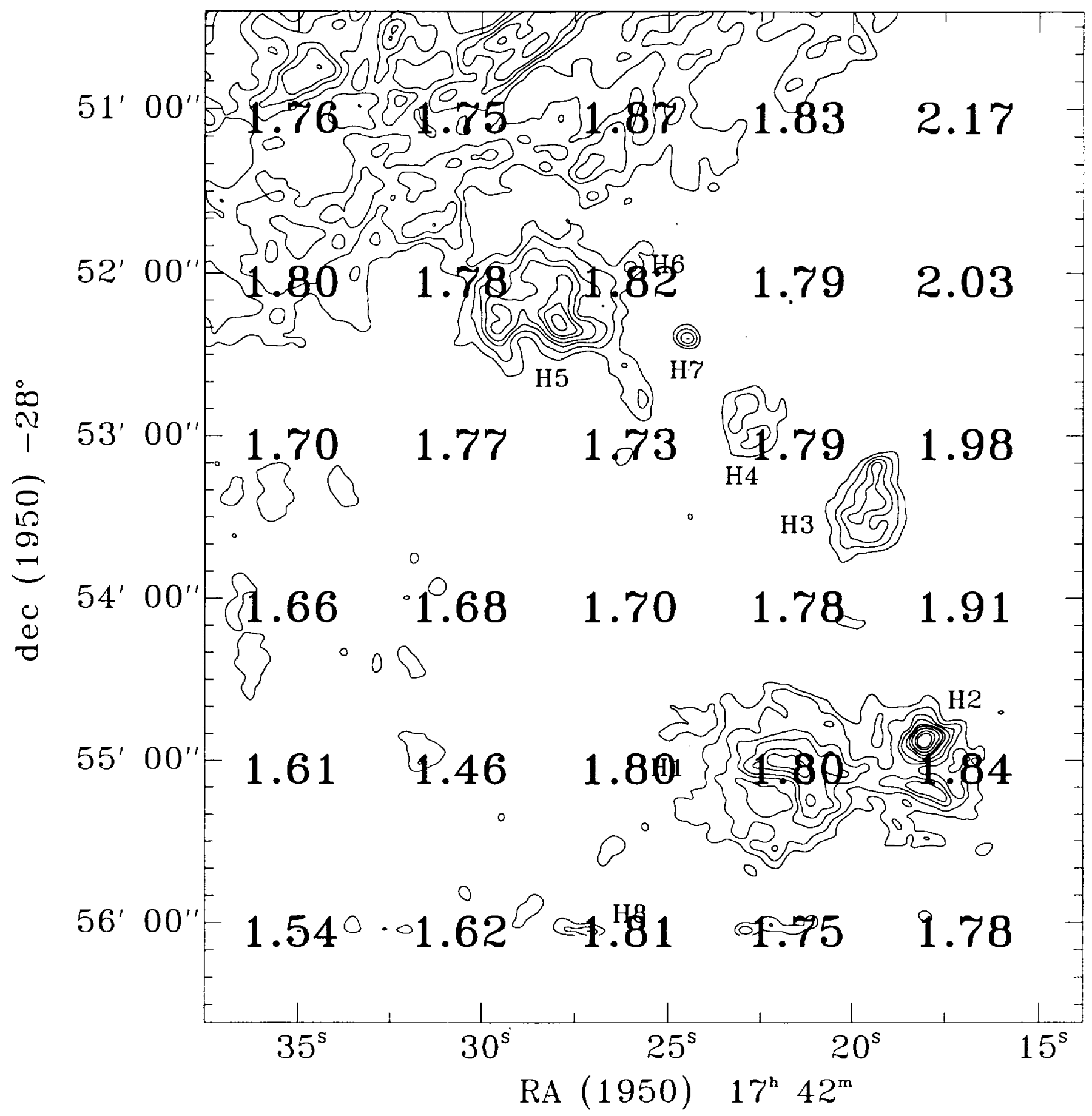




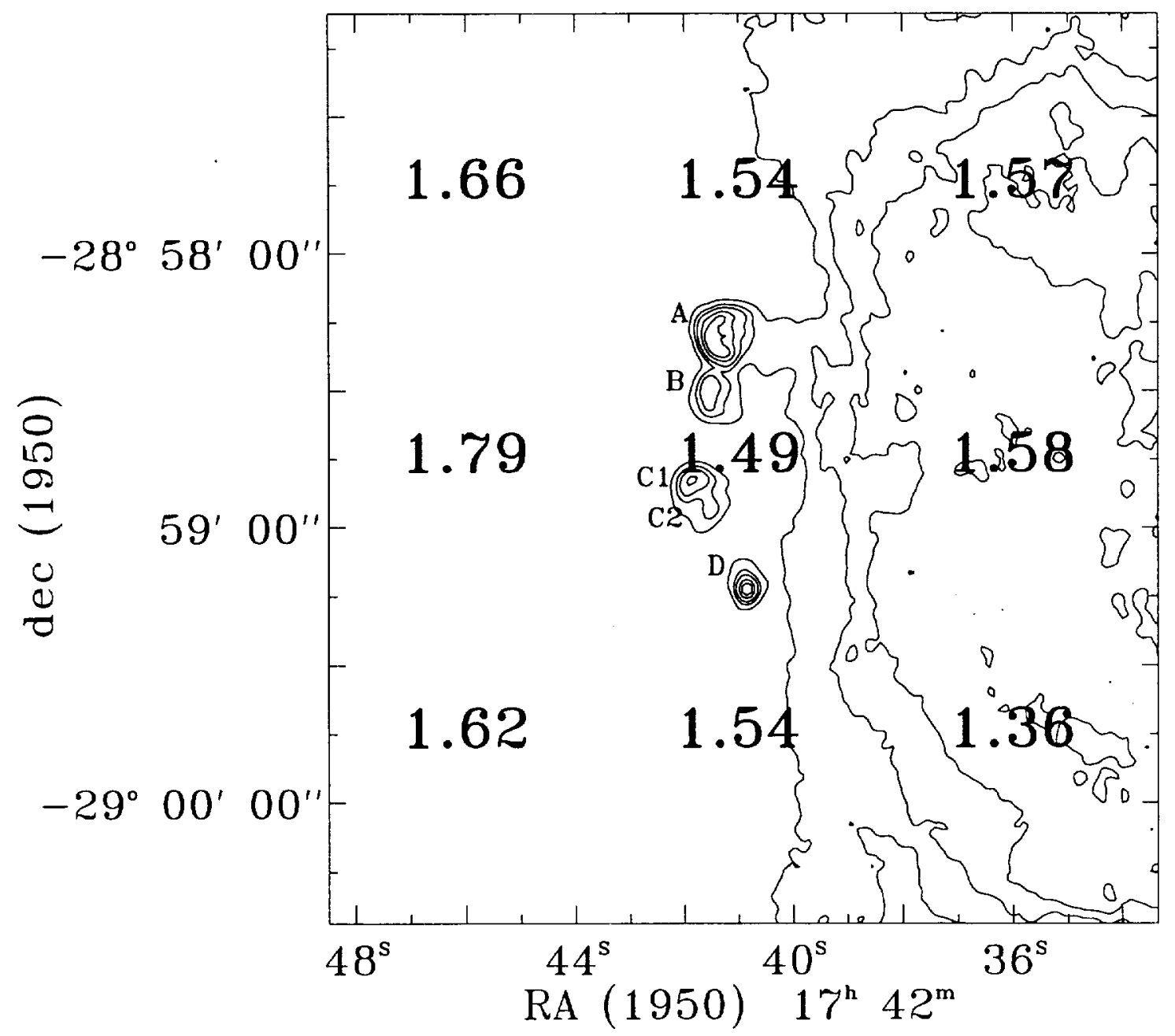

